\title{
Review Article \\ Bougainvillea Genus: A Review on Phytochemistry, Pharmacology, and Toxicology
}

\author{
Rodolfo Abarca-Vargas $(\mathbb{D}$ and Vera L. Petricevich \\ Facultad de Medicina de la Universidad Autónoma del Estado de Morelos (UAEM), Calle Leñeros, \\ Esquina Iztaccíhuatl s/n. Col. Volcanes, Cuernavaca, Morelos, México, C.P. 62350, Mexico
}

Correspondence should be addressed to Vera L. Petricevich; vera.petricevich@uaem.mx

Received 20 February 2018; Revised 17 April 2018; Accepted 21 May 2018; Published 24 June 2018

Academic Editor: Wen-yi Kang

Copyright (C) 2018 Rodolfo Abarca-Vargas and Vera L. Petricevich. This is an open access article distributed under the Creative Commons Attribution License, which permits unrestricted use, distribution, and reproduction in any medium, provided the original work is properly cited.

\begin{abstract}
This review discusses the current knowledge of the phytochemistry and in vitro and in vivo evaluations carried out using the extracts and, where appropriate, the main active components isolated from the genus Bougainvillea. Out of 18 species, most phytochemical, pharmacological, and toxicological studies focused on four species with different cultivars and one hybrid. Some plants are used for the treatment of various health disorders. Numerous phytochemical investigations of plants in this genus confirm the presence of aliphatic hydrocarbons, fatty acids, fatty alcohols, volatile compounds, phenolic compounds, peltogynoids, flavonoids, phytosterols, terpenes, carbohydrates, and betalains. Various studies have confirmed that these extracts or active substances that were isolated from the genus Bougainvillea have multiple pharmacological activities. Some species of Bougainvillea have emerged as sources of traditional medicine in human health. More studies of the phytochemical, pharmacological, and toxicological properties and their mechanisms of action, safety, and efficacy in all Bougainvillea species, cultivars, and hybrids are advisable for future research.
\end{abstract}

\section{Introduction}

The genus Bougainvillea is a very widespread group throughout the world. It belongs to the family Nyctaginaceae and, according to the "The Plant List", contains approximately 18 species (B. berberidifolia, B. buttiana, B. campanulata, B. glabra, B. herzogiana, B. infesta, B. lehmanniana, B. lehmannii, B. malmeana, B. modesta, B. pachyphylla, B. peruviana, B. pomacea, B. praecox, B. spectabilis, B. spinosa, B. stipitata, and B. trollii) [1]. Only four species (B. buttiana, B. glabra, B. spectabilis, and B. peruviana) are commercially exploited [2]. However, there are also more than 100 cultivars and three hybrids, the latter not yet recognized.

The objectives of this review are to provide updated and complete information on the distribution, phytochemical, pharmacological, and toxicity research of Bougainvillea species, to identify their therapeutic potential and to direct future research opportunities. The most relevant data were searched using the keyword "Bougainvillea" in "Google Scholar", "PubMed", "ScienceDirect", "Scopus", and "Web of
Science". The taxonomy was validated using the "The Plant List".

\section{Ethnobotany}

\subsection{Taxonomical Classification}

Kingdom: Plantae

Subkingdom: Tracheobionta

Superdivision: Spermatophyta

Division: Magnoliophyta

Class: Magnoliopsida

Subclass: Caryophyllidae

Order: Caryophyllales

Family: Nyctaginaceae

Genus: Bougainivillea $[3,4]$ 
2.2. Botanical Characterization and Distribution. The genus Bougainvillea is endemic to South America and was firstly reported in Brazil in 1778 before being introduced to Europe, by French military commander Louis Antoine de Bougainville [2]. They are bushes spread in vines or small trees. They also possess stems with internodes and with straight or slightly curved thorns. The leaves are petiolate, elliptical, or wider towards the base. The bracts and flowers are presented in different colours, depending on the species, cultivars, or hybrid. They bloom throughout the year [5].

Botanical textbooks say that some species of the genus Bougainvillea are distributed worldwide and without being specific to any single place, species, cultivars, or hybrids. Based on these results, a more exhaustive analysis was performed in the scientific literature. In this review we highlight a more reliable update with respect to the distribution based on the scientific literature or scientific research works.

(i) B. buttiana: this species was found in India [6], Mexico [7], and Thailand [8].

(ii) B. glabra: it was found in Italy [9], Spain, France [10], Bangladesh [11], India [12], China [13], Egypt [14], Israel [15], Thailand [16], Philippines [17], Madagascar [5], Nigeria [18], Hawaii [2], Bolivia, Colombia, Costa Rica, Cuba, Ecuador, El Salvador, United States of America, Guatemala, Honduras, Virgin Islands, Mexico, Nicaragua, Puerto Rico, Dominican Republic, Venezuela [5], and Brazil [19].

(iii) B. spectabilis: this species was reported in Nigeria [20], Bahamas, Bolivia, Colombia, Costa Rica, Ecuador, Guatemala, Honduras, Jamaica, Mexico, Panama, Puerto Rico, Dominican Republic, Tanzania, Trinidad and Tobago [5], India [21], Montenegro [22], Pakistan [23], Australia, Brazil [19], and Vietnam [24].

(iv) B. spinosa: this plant species is a very poorly documented plant species, but it was reported in Argentina [25].

(v) B. peruviana: this plant is scarce and only noted in the scientific literature in China [13], India [19], and Peru [2].

2.3. Bougainvillea Hybrids. Since the beginning of its commercialization, producers have sought to make hybrids of the genus Bougainvillea species. They have produced three recognized hybrids with striking characteristics: $B . x$ spectoperuviana, B. x spectoglabra, and B. glabra peruviana (B. $x$ buttiana).

(i) B. $x$ spectoperuviana: the first hybrid has no reports in the scientific literature of registration sites or scientific research works according to the databases consulted.

(ii) B. $\boldsymbol{x}$ spectoglabra: this plant is a hybrid of B. spectabilis and $B$. peruviana and it was only reported in China [13].

(iii) B. glabraperuviana or B. $x$ buttiana: this plant is a hybrid of $B$. glabra and B. peruviana, and it was identified and reported in Mexico [26], as well as in
India, England [19], and China [13]. This plant was thoroughly studied by our working group, and those results are described below.

There is no other current scientific information regarding the location, medical uses, phytochemical profile, or pharmacological or toxicological properties for other species or hybrids.

2.4. Synonyms. In the different countries where the Bougainvillea are found other popular names were attributed: Buganvilla (Spain), Bugambilia (Mexico, Guatemala, Cuba, and Philippines), Pokok bunga kertas (Malaysia), Napoleón (Honduras), Veranera (Colombia, Nicaragua, El salvador, Costa Rica, and Panama), Trinitaria (Colombia, Panama, Puerto Rico, Dominican Republic, and Venezuela), Santa Rita (Argentina, Bolivia, Brazil, Paraguay, and Uruguay), Primavera, Tres-Marias, Sempre-lustrosa, Santa-rita, Ceboleiro, Roseiro, Roseta, Riso, Pataguinha, Pau-de-roseira, and Flor-depapel (Brazil) or Papelillo (Northern Peru) [3].

2.5. Traditional Medical Use. In traditional medicine the species B. buttiana, B. glabra, and B. spectabilis are indicated for the treatment of coughing [5] and pertussis [7]. B. glabra is recommended for asthma [27], bronchitis, and dysentery. In a small number of cases, it is indicated for stomach pain, rust, pimples, and blackheads. B. spectabilis is also used in other respiratory conditions, including snoring or lung pain, flu, and bronchitis [7]. There are no studies described in the literature regarding the traditional use of the other species and hybrids of Bougainvillea in medicine. However, the hybrid B. $x$ buttiana was confused with B. buttiana since both are distributed and reported in Morelos, Mexico, and both are used to treat cough and whooping cough $[5,28,29]$.

\section{Phytochemistry}

The chemical constituents of the genus Bougainvillea have been extensively studied since 1970 [9, 37]. The phytochemical analyses were carried out to identify different kinds of components using extracts of different polarities from stems, leaves, or bracts with or without flowers, bark stems, and roots of the species. It has been possible to isolate, identify, and elucidate chemical compounds for species or hybrids. The Marvin program was used to draw the structures of chemical compounds [73].

3.1. Aliphatic Hydrocarbons. In Bougainvillea genus the presence of aliphatic hydrocarbons including alkanes, alkenes, and cycloalkanes has been described. For ethanolic extracts from bracts with flowers from $B . x$ buttiana seven of these compounds were found and identified. The presence of this type of compounds, B. $x$ buttiana, could be considered as an alternative source of energy (Table 1 and Figure 1).

3.2. Fatty Acids and Fatty Alcohols. Fatty acids and fatty alcohols are very common compounds in plants, especially in aerial parts. For the genus of Bougainvillea, the presence of 13 of these compounds was verified. Eight compounds were 
TABLE 1: Alkanes, alkenes, and cycloalkanes from genus Bougainvillea.

\begin{tabular}{lclll}
\hline No. & Compound's name & Species & Parts used & Reference \\
\hline$(\mathbf{1})$ & Pentacosane & B. $x$ buttiana & Bracts with flowers & [26] \\
$(2)$ & Heptacosane & B. $x$ buttiana & Bracts with flowers & Bracts with flowers \\
$(3)$ & Nonacosane & B. $x$ buttiana & Bracts with flowers & {$[26]$} \\
$(4)$ & 9-Octylhexacosane & B. $x$ buttiana & Bracts with flowers & {$[30]$} \\
$(5)$ & 1-Nonadecene & B. $x$ buttiana & Bracts with flowers & {$[26]$} \\
$(6)$ & 1-Hexacosene & B. $x$ buttiana & Bracts with flowers & {$[26]$} \\
\hline
\end{tabular}

TABLE 2: Fatty acid and fatty alcohols from genus Bougainvillea.

\begin{tabular}{|c|c|c|c|c|}
\hline No. & Compound's name & Species & Parts used & Reference \\
\hline (8) & 2-Methylpropanoic acid & B. spectabilis & Leaves and branches & [22] \\
\hline (9) & Dodecanoic acid & B. spectabilis & Leaves and branches & {$[22]$} \\
\hline (10) & Tetradecanoic acid & B. $x$ buttiana & Bracts with flowers & {$[26]$} \\
\hline (11) & Hexadecanoic acid & B. $x$ buttiana & Bracts with flowers & {$[26,30]$} \\
\hline (12) & Octadecanoic acid & B. $x$ buttiana & Bracts with flowers & {$[26]$} \\
\hline (13) & 9-Octadecenoic acid (E)- & B. $x$ buttiana & Bracts with flowers & {$[26]$} \\
\hline (14) & 9,12-Octadecadienoic acid (Z,Z) & B. $x$ buttiana & Bracts with flowers & {$[26]$} \\
\hline (15) & $n$-Octacos-9-enoic acid & B. spectabilis & Roots & [31] \\
\hline$(16)$ & 1,2-Dipalmitoleoyl glyceryl phosphate & B. spectabilis & Roots & [31] \\
\hline (17) & 1-Triacontanol & B. $x$ buttiana & Bracts with flowers & {$[26]$} \\
\hline (18) & 1-Dotriacontanol & B. $x$ buttiana & Bracts with flowers & [26] \\
\hline (19) & $n$-Hentriacontanol & B. spectabilis & Roots & {$[31]$} \\
\hline (20) & 1,30-Triacontanediol & B. $x$ buttiana & Bracts with flowers & [26] \\
\hline
\end{tabular}

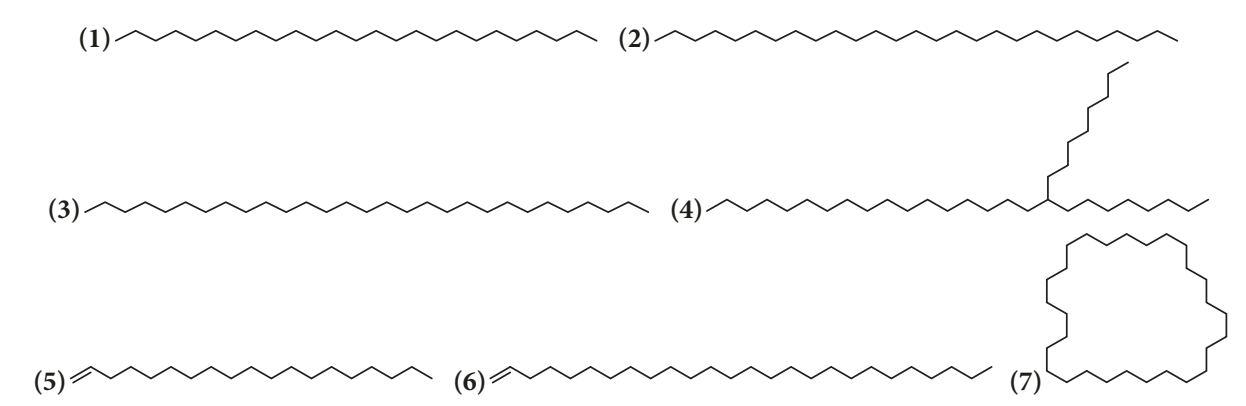

FIgURE 1: Structure of alkanes, alkenes, and cycloalkanes from genus Bougainvillea.

identified in ethanolic extracts from the bracts with flowers from $B . x$ buttiana and 5 in ethanolic extracts of the leaves, branches, and roots from $B$. spectabilis (Table 2 and Figure 2).

3.3. Volatile Compounds. Volatile compounds are compounds that are commonly found in the plant kingdom. Their chemical structures contain some functional groups, including aldehydes, ketones, phenols, oxides, esters, and alcohols. In the leaves and branches of B. spectabilis ethanol extract were identified 35 of these compounds. In ethanol, ethanol:water, and ethyl acetate extracts of bracts with flowers of $B . x$ buttiana the presence of 9 of these compounds was identified. Only one compound was similar as is the case of the ethyl hexadecanoate compound observed in both extracts B. $x$ buttiana and B. spectabilis (Table 3 and Figure 3).
3.4. Phenolic Compounds. Phenolic compounds are also widely distributed in the plant kingdom. Fourteen of these compounds have been identified. In the ethanolic extract of bracts with flowers from $B . x$ buttiana researchers identified 4 of these compounds. In ethanolic extracts of flowers from B. glabra there were 11 phenolic compounds. Compounds $\mathbf{7 6}$ and 77 are not common in plants; however, their presence is reported in hybrid B. $x$ buttiana (Table 4 and Figure 4).

3.5. Peltogynoids and Flavonoids. The peltogynoids are restricted in their distribution. In extracts of stem bark from $B$. spectabilis were identified eight peltogynoids. The flavonoids, however, are a group of compounds widely distributed in the plant kingdom, 21 compounds have been identified in $B$. glabra and B. spectabilis (Table 5 and Figure 5). 


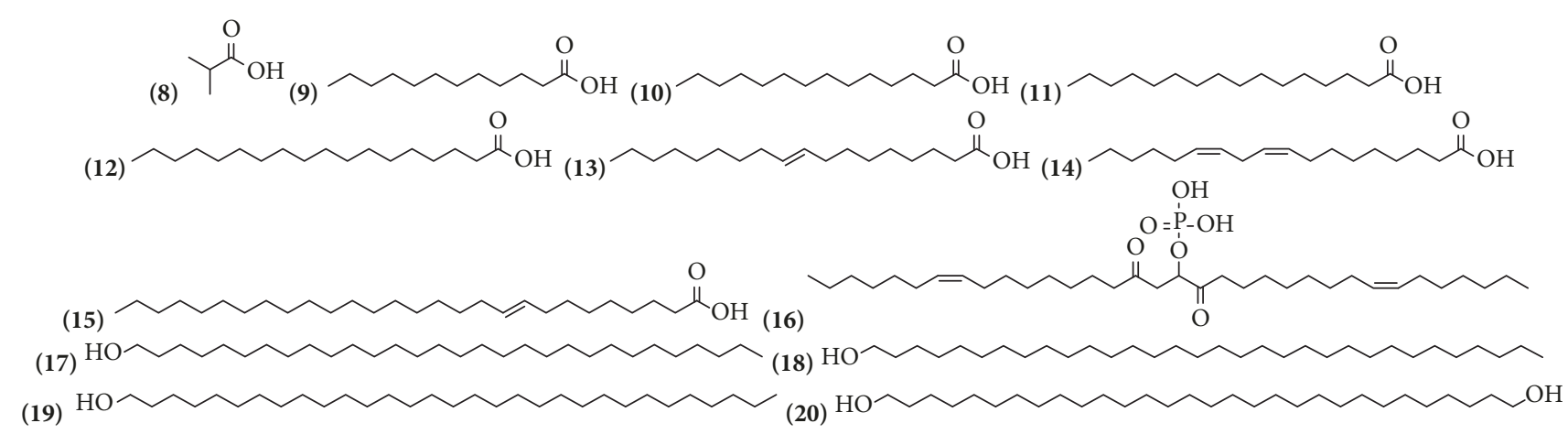

FIGURE 2: Structure of fatty acid and fatty alcohols from genus Bougainvillea.

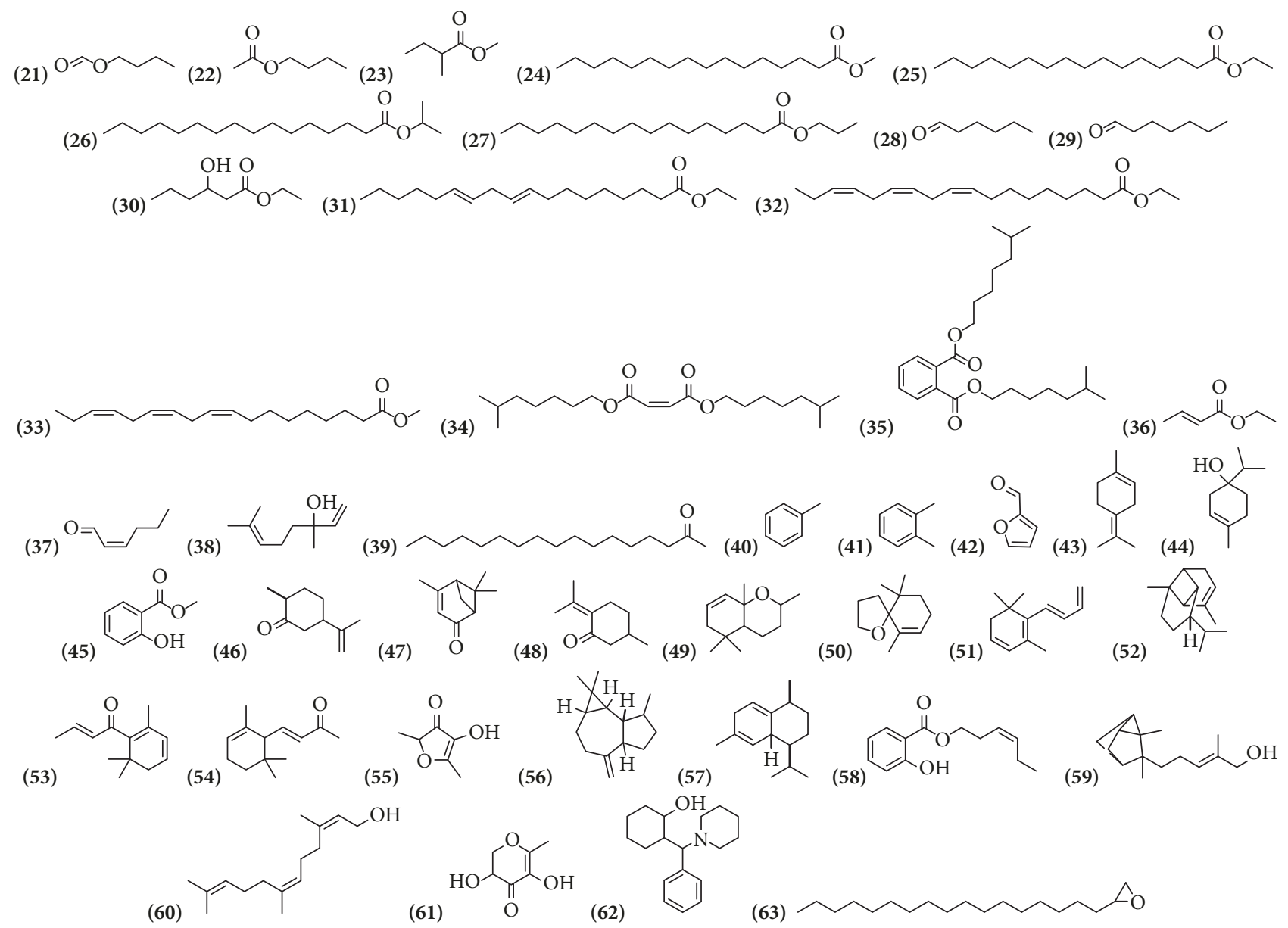

FIGURE 3: Structure of volatile compounds from genus Bougainvillea.

3.6. Phytosterols, Terpenes, and Carbohydrates. Carbohydrates are chemical compounds that mainly derive from the primary metabolism of vegetables. Sterols and terpenes are secondary metabolites. Out of thirteen compounds identified in the genus Bougainvillea, 6 of them were identified from the ethanolic extracts of bracts with flowers from B. $x$ buttiana. Four different compounds were identified from extracts of leaves and bracts from B. glabra. Three in B. spectabilis in stem bark, leaves, and branches. Only one compound was similar as is the case of the squalene compound observed in both extracts B. glabra and B. $x$ buttiana (Table 6 and Figure 6).

3.7. Betalains. Betalains are vacuolar pigments containing a nitrogenous ring, a ring which is characteristic of the order Caryophyllales. Sixteen of these compounds were identified in bract extracts from the case of extracts of B. glabra and 2 were found in extracts from B. Mrs. Butt (Table 7 and Figure 7). 
TABLE 3: Volatile compounds from genus Bougainvillea.

\begin{tabular}{|c|c|c|c|c|}
\hline No. & Compound's name & Species & Parts used & Reference \\
\hline$(21)$ & Butyl formate & B. spectabilis & Leaves and branches & {$[22]$} \\
\hline (22) & Butyl acetate & B. spectabilis & Leaves and branches & {$[22]$} \\
\hline (23) & Methyl 2-methylbutanoate & B. spectabilis & Leaves and branches & {$[22]$} \\
\hline (24) & Methyl hexadecanoate & B. spectabilis & Leaves and branches & {$[22]$} \\
\hline \multirow[t]{2}{*}{ (25) } & Ethyl hexadecanoate & B. spectabilis & Leaves and branches & {$[22]$} \\
\hline & & B. $x$ buttiana & Bracts with flowers & {$[26]$} \\
\hline (26) & Isopropyl palmitate & B. $x$ buttiana & Bracts with flowers & {$[30]$} \\
\hline$(27)$ & Propyl hexadecanoate & B. spectabilis & Leaves and branches & {$[22]$} \\
\hline (28) & Hexanal & B. spectabilis & Leaves and branches & {$[22]$} \\
\hline (29) & Heptanal & B. spectabilis & Leaves and branches & {$[22]$} \\
\hline (30) & Ethyl 3-hydroxy-hexanoate & B. spectabilis & Leaves and branches & {$[22]$} \\
\hline$(31)$ & 9,12-Octadecadienoic acid, ethyl ester & B. $x$ buttiana & Bracts with flowers & {$[26]$} \\
\hline (32) & 9,12,15-Octadecatrienoic acid, ethyl ester, $(\mathrm{Z}, \mathrm{Z}, \mathrm{Z})$ & B. $x$ buttiana & Bracts with flowers & {$[26]$} \\
\hline (33) & Methyl linolenate & B. spectabilis & Leaves and branches & {$[22]$} \\
\hline (34) & Diisooctyl maleate & B. $x$ buttiana & Bracts with flowers & {$[30]$} \\
\hline (35) & 1,2-Benzenedicarboxylic acid, diisooctyl ester & B. $x$ buttiana & Bracts with flowers & {$[30]$} \\
\hline$(36)$ & Ethyl (E)-crotonate & B. spectabilis & Leaves and branches & {$[22]$} \\
\hline$(37)$ & (Z)-2-Hexenal & B. spectabilis & Leaves and branches & {$[22]$} \\
\hline$(38)$ & Linaool & B. spectabilis & Leaves and branches & {$[22]$} \\
\hline (39) & 2-Heptadecanone & B. spectabilis & Leaves and branches & {$[22]$} \\
\hline (40) & Toluene & B. spectabilis & Leaves and branches & {$[22]$} \\
\hline (41) & $O$-xylene & B. spectabilis & Leaves and branches & {$[22]$} \\
\hline (42) & 2-Furfural & B. spectabilis & Leaves and branches & {$[22]$} \\
\hline (43) & Terpinolene & B. spectabilis & Leaves and branches & {$[22]$} \\
\hline$(44)$ & Terpinen-4-ol & B. spectabilis & Leaves and branches & {$[22]$} \\
\hline (45) & Methyl salicylate & B. spectabilis & Leaves and branches & {$[22]$} \\
\hline (46) & Trans-dihydrocarvone & B. spectabilis & Leaves and branches & {$[22]$} \\
\hline (47) & Verbenone & B. spectabilis & Leaves and branches & {$[22]$} \\
\hline$(48)$ & Pulegone & B. spectabilis & Leaves and branches & {$[22]$} \\
\hline (49) & Dihydroedulan II & B. spectabilis & Leaves and branches & {$[22]$} \\
\hline (50) & Theaspirane B & B. spectabilis & Leaves and branches & {$[22]$} \\
\hline$(51)$ & Dehydroionene & B. spectabilis & Leaves and branches & {$[22]$} \\
\hline$(52)$ & $\alpha$-copaene & B. spectabilis & Leaves and branches & {$[22]$} \\
\hline (53) & (E)- $\beta$-damascenone & B. spectabilis & Leaves and branches & {$[22]$} \\
\hline (54) & $\alpha$-(E)-Ionone & B. spectabilis & Leaves and branches & {$[22]$} \\
\hline (55) & 2,5-Dimethyl-4-hydroxy-3(2H)-furanone & B. spectabilis & Leaves and branches & {$[22]$} \\
\hline$(56)$ & Aromadendrene & B. spectabilis & Leaves and branches & {$[22]$} \\
\hline$(57)$ & Cadina-1.4-diene & B. spectabilis & Leaves and branches & {$[22]$} \\
\hline$(58)$ & (Z)-3-Hexenyl salicylate & B. spectabilis & Leaves and branches & {$[22]$} \\
\hline$(59)$ & $\alpha$-santalol & B. spectabilis & Leaves and branches & {$[22]$} \\
\hline$(60)$ & $(\mathrm{Z}, \mathrm{Z})$-farnesol & B. spectabilis & Leaves and branches & {$[22]$} \\
\hline$(61)$ & 4H-Pyran-4-one, 2,3-dihydro-3,5-dihydroxy-6-methyl & B. $x$ buttiana & Bracts with flowers & {$[26]$} \\
\hline$(62)$ & 2-(Phenyl-piperidin-1-yl-methyl)-cyclohexanol & B. $x$ buttiana & Bracts with flowers & {$[26]$} \\
\hline$(63)$ & Oxirane, heptadecyl- & B. $x$ buttiana & Bracts flowers & {$[26]$} \\
\hline
\end{tabular}

\section{Pharmacological Activity}

Four species with different cultivars and one hybrid of Bougainvillea have been reported in traditional medicine. A more general view of pharmacological investigations on various crude extracts and isolated chemical compounds of these species is described below.

4.1. Analgesic. The analgesic activity was described in two species of B. glabra [48] and B. x buttiana; in both cases 
TABle 4: Phenolic compounds from genus Bougainvillea.

\begin{tabular}{|c|c|c|c|c|}
\hline No. & Compound's name & Species & Parts used & Reference \\
\hline$(64)$ & $\begin{array}{c}\text { Ethanone, } \\
\text { 1-(2-hydroxy-5-methylphenyl) }\end{array}$ & B. $x$ buttiana & Bracts with flowers & {$[26]$} \\
\hline$(65)$ & Gallic acid & B. glabra & Flowers & {$[16]$} \\
\hline$(66)$ & Syringic acid & B. glabra & Flowers & {$[16]$} \\
\hline (67) & p-Hydroxybenzoic acid & B. glabra & Flowers & {$[16]$} \\
\hline$(68)$ & Protocatechuic acid & B. glabra & Flowers & {$[16]$} \\
\hline (69) & Vanillic acid & B. glabra & Leaves & {$[32]$} \\
\hline (70) & Ferulic acid & B. glabra & Flowers & [16] \\
\hline$(71)$ & Sinapic acid & B. glabra & Flowers & [16] \\
\hline \multirow[t]{2}{*}{ (72) } & $\begin{array}{l}\text { 2-Propenoic acid, } \\
\text { 3-(2-hydrophenyl)-(E)- }\end{array}$ & B. glabra & Flowers & {$[16]$} \\
\hline & & B. $x$ buttiana & Bracts with flowers & {$[30]$} \\
\hline (73) & Caffeic acid & B. glabra & Flowers & {$[16]$} \\
\hline (74) & Coumaric acid & B. glabra & Leaves & {$[32]$} \\
\hline (75) & Chlorogenic acid & B. glabra & Flowers & [16] \\
\hline$(76)$ & 2,4-Di-tert-butylphenol & B. $x$ buttiana & Bracts with flowers & {$[26]$} \\
\hline (77) & $\begin{array}{l}\text { Naphthalene, 3,4-dihydro-1,8-bis } \\
\text { (trimethylsilyloxy) }\end{array}$ & B. $x$ buttiana & Bracts with flowers & {$[26]$} \\
\hline
\end{tabular}

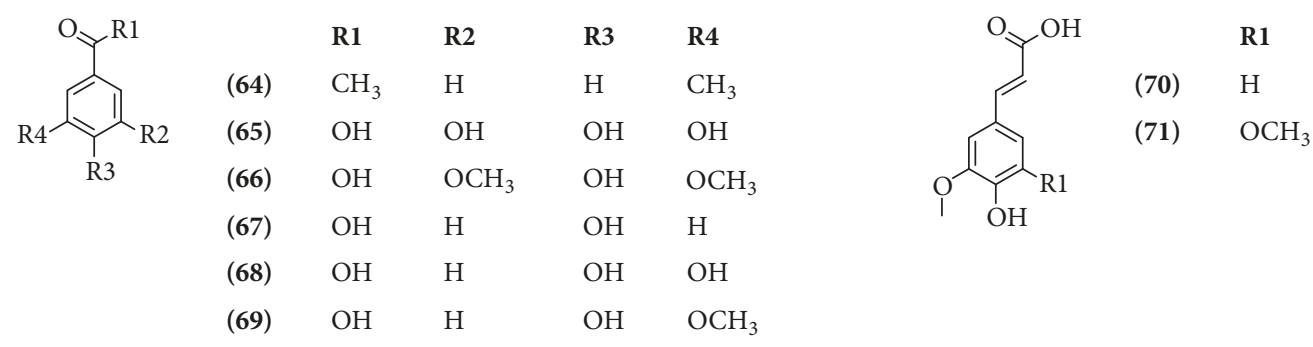

$\begin{array}{lllll}\mathbf{( 7 2 )} & \mathrm{H} & \mathrm{H} & \mathrm{OH} \\ & (\mathbf{7 3 )} & \mathrm{OH} & \mathrm{OH} & \mathrm{H} \\ & (74) & \mathrm{OH} & \mathrm{H} & \mathrm{H}\end{array}$<smiles>CC(C)(C)c1ccc(O)c(C(C)(C)C)c1</smiles>

FIgURE 4: Structure of phenolic compounds from genus Bougainvillea.

the oral administration was evaluated $[29,49]$. For methanol extracts of $B$. glabra, the maximum percentage of analgesia effect obtained using the tail method in male Wistar rats was $79.88 \%$ [48]. For the ethanol extracts of B. $x$ buttiana (var. Orange), the analgesic effect was studied in female CD1 mice using the acetic acid and formalin methods. For the acetic acid method, the analgesia percentage was 95.65\%, while, for formalin method, the extract showed inhibition in both phases [49]. In another study, the analgesic effect of the $B$. $x$ buttiana (var. Rose) ethanol extract was determined after oral administration in BALB/c mice using the acetic acid, tail immersion, and formalin models. For all of the methods used, the extract showed a potent analgesic effect [29].
4.2. Anti-Inflammatory. A significant anti-inflammatory activity was obtained in male Wistar rats orally treated with methanol extract of leaves from B. glabra [48].

The leaves from $B$. spectabilis were extracted with different solvents, including acetone, alcohol, chloroform, petroleum ether, and chloroform:water. The models used were the oedema induced by carrageenin and the granuloma model induced by cotton pellet, in male Wistar rats. The results indicated that the oral administration in rats with ethanol extract reduced the oedema induced by both methods [50]. In other experiments, the oral administration of methanol extract of leaf from B. spectabilis was performed and evaluated in Swiss mice using the method of induction oedema with 
TABLE 5: Peltogynoids and flavonoids from genus Bougainvillea.

\begin{tabular}{|c|c|c|c|c|}
\hline No. & Compound's name & Species & Parts used & Reference \\
\hline (78) & Bougainvinone A & B. spectabilis & Stem bark & {$[24]$} \\
\hline (79) & Bougainvinone $\mathrm{B}$ & B. spectabilis & Stem bark & {$[24]$} \\
\hline$(80)$ & Bougainvinone $\mathrm{C}$ & B. spectabilis & Stem bark & {$[24]$} \\
\hline$(81)$ & Bougainvinone D & B. spectabilis & Stem bark & {$[24]$} \\
\hline$(82)$ & Bougainvinone $\mathrm{E}$ & B. spectabilis & Stem bark & {$[24]$} \\
\hline (83) & Bougainvinone $\mathrm{F}$ & B. spectabilis & Stem bark & {$[24]$} \\
\hline (84) & Bougainvinone $\mathrm{G}$ & B. spectabilis & Stem bark & {$[24]$} \\
\hline (85) & Bougainvinone $\mathrm{H}$ & B. spectabilis & Stem bark & {$[24]$} \\
\hline$(86)$ & Bougainvinone I & B. spectabilis & Stem bark & {$[33]$} \\
\hline$(87)$ & Bougainvinone J & B. spectabilis & Stem bark & [33] \\
\hline (88) & Bougainvinone $\mathrm{K}$ & B. spectabilis & Stem bark & {$[33]$} \\
\hline (89) & Bougainvinone L & B. spectabilis & Stem bark & {$[33]$} \\
\hline (90) & Bougainvinone $\mathrm{M}$ & B. spectabilis & Stem bark & {$[33]$} \\
\hline (91) & 5,7,3', $4^{\prime}$-Tetrahydroxy-3-methoxy-6,8-dimethylflavone & B. spectabilis & Stem bark & {$[33]$} \\
\hline$(92)$ & 5,7,4'-Trihydroxy-3-methoxy-6,8-dimethylflavone & B. spectabilis & Stem bark & {$[33]$} \\
\hline (93) & Rutin & B. glabra & Flowers & {$[16]$} \\
\hline (94) & Isovitexin & B. glabra & Leaves & {$[14]$} \\
\hline (95) & Vitexin & B. glabra & Leaves & {$[14]$} \\
\hline (96) & Apigenin & B. glabra & Flowers & {$[16]$} \\
\hline (97) & Kaempferol & B. glabra & Flowers & {$[16]$} \\
\hline (98) & Chrysoeriol & B. glabra & Leaves & {$[14]$} \\
\hline (99) & Luteolin & B. glabra & Leaves & {$[14]$} \\
\hline$(100)$ & Myricetin & B. glabra & Flowers & [16] \\
\hline (101) & Quercetin & B. glabra & Flowers & {$[16]$} \\
\hline (102) & Quercitrin & B. glabra & Stem bark & [34] \\
\hline$(103)$ & $\begin{array}{c}\text { Quercetin 3-O- } \alpha \text {-L-(rhamnopyranosyl })(1 \rightarrow 6)-[\alpha \text {-L-rhamnopyranosyl }(1 \rightarrow 2)] \text { - } \\
\beta \text {-D-galactopyranoside }\end{array}$ & B. glabra & Bracts & {$[35]$} \\
\hline$(104)$ & $\begin{array}{l}\text { Quercetin 3-O- } \alpha \text {-L-(4-caffeoylrhamnopyranosyl })(1 \rightarrow 6) \text { - }[\alpha \text {-L- } \\
\text { rhamnopyranosyl }(1 \rightarrow 2)]-\beta \text {-D-galactopyranoside }\end{array}$ & B. glabra & Bracts & {$[35]$} \\
\hline$(105)$ & $\begin{array}{c}\left.\text { Luteolin-7-O-[2" }-O-\left(5^{\prime \prime \prime}-O \text {-feruloyl }\right)-\beta-\mathrm{D} \text {-apiofuranosyl }\right]-\beta-\mathrm{D}- \\
\text { glucopyranoside }\end{array}$ & B. glabra & Leaves & [14] \\
\hline$(106)$ & $2^{\prime}$-Hydroxydemethoxymatteucinol & B. spectabilis & Stem bark & {$[33]$} \\
\hline
\end{tabular}

TABLE 6: Phytosterol, terpenes, and carbohydrates from genus Bougainvillea.

\begin{tabular}{|c|c|c|c|c|}
\hline No. & Compound's name & Species & Parts used & Reference \\
\hline (107) & Chondrillasterol & B. $x$ buttiana & Bracts with flowers & {$[26]$} \\
\hline (108) & Stigmasta-5,22-dien-3-ol & B. $x$ buttiana & Bracts with flowers & {$[26]$} \\
\hline (109) & Stigmasterol & B. glabra & Leaves & {$[32]$} \\
\hline (110) & $\beta$-sitosterol & B. spectabilis & Stem bark & {$[34]$} \\
\hline (111) & Stigmast-7-en-3-ol, $(3 \beta, 5 \alpha)$ & B. $x$ buttiana & Bracts with flowers & {$[26]$} \\
\hline (112) & Momordin IIc & B. glabra & Bracts & {$[35]$} \\
\hline \multirow[t]{2}{*}{ (113) } & Squalene & B. glabra & Leaves & {$[32]$} \\
\hline & & B. $x$ buttiana & Bracts with flowers & {$[32]$} \\
\hline (114) & Isophytol & B. spectabilis & Leaves and branches & {$[22]$} \\
\hline (115) & Phytol & B. spectabilis & Leaves and branches & {$[22]$} \\
\hline (116) & Geranylgeraniol & B. glabra & Leaves & {$[32]$} \\
\hline (117) & $\alpha$-Tocopherol & B. $x$ buttiana & Bracts with flowers & {$[26]$} \\
\hline (118) & Pinitol & B. spectabilis & Leaves & {$[36]$} \\
\hline (119) & 3-O-Methyl-D-glucose & B. $x$ buttiana & Bracts with flowers & {$[26]$} \\
\hline
\end{tabular}


<smiles>[R2]c1cc([R1])c2c(c1)COc1c-2oc2c(C)c([R4])c(C)c([R3])c2c1=O</smiles>

$\begin{array}{lllll} & \mathbf{R} 1 & \mathbf{R} 2 & \mathbf{R} 3 & \mathbf{R} 4 \\ \mathbf{( 7 8 )} & \mathrm{OH} & \mathrm{H} & \mathrm{OCH}_{3} & \mathrm{OCH}_{3} \\ \mathbf{( 7 9 )} & \mathrm{OH} & \mathrm{H} & \mathrm{OH} & \mathrm{OCH}_{3} \\ \mathbf{( 8 0 )} & \mathrm{OH} & \mathrm{H} & \mathrm{OH} & \mathrm{OH} \\ (\mathbf{8 1}) & \mathrm{H} & \mathrm{H} & \mathrm{OH} & \mathrm{OH} \\ (\mathbf{8 2}) & \mathrm{H} & \mathrm{H} & \mathrm{OCH}_{3} & \mathrm{OH} \\ \mathbf{( 8 3 )} & \mathrm{H} & \mathrm{H} & \mathrm{OCH}_{3} & \mathrm{OCH} \\ (\mathbf{8 4}) & \mathrm{H} & \mathrm{OCH}_{3} & \mathrm{OH} & \mathrm{OH} \\ (\mathbf{8 5}) & \mathrm{H} & \mathrm{OCH}_{3} & \mathrm{OCH}_{3} & \mathrm{OH}\end{array}$<smiles>[R1]c1cc([R1])c(-c2oc3c(C)c(O)c(C)c([R3])c3c(=O)c2OC)c([R1])c1[R3]</smiles>

$\begin{array}{llllll} & \mathbf{R} 1 & \mathbf{R 2} & \mathbf{R} 3 & \mathbf{R} 4 & \mathbf{R} 5 \\ \mathbf{( 8 6 )} & \mathrm{OH} & \mathrm{H} & \mathrm{H} & \mathrm{H} & \mathrm{OH} \\ \mathbf{( 8 7 )} & \mathrm{OH} & \mathrm{H} & \mathrm{H} & \mathrm{CH}_{2} \mathrm{OH} & \mathrm{OH} \\ \mathbf{( 8 8 )} & \mathrm{H} & \mathrm{H} & \mathrm{H} & \mathrm{CH}_{2} \mathrm{OH} & \mathrm{OCH}_{3} \\ \mathbf{( 8 9 )} & \mathrm{H} & \mathrm{OH} & \mathrm{H} & \mathrm{H} & \mathrm{OCH}_{3} \\ \mathbf{( 9 0 )} & \mathrm{H} & \mathrm{H} & \mathrm{H} & \mathrm{H} & \mathrm{OCH}_{3} \\ \mathbf{( 9 1 )} & \mathrm{H} & \mathrm{OH} & \mathrm{OH} & \mathrm{H} & \mathrm{OH} \\ \mathbf{( 9 2 )} & \mathrm{H} & \mathrm{OH} & \mathrm{H} & \mathrm{H} & \mathrm{OH}\end{array}$<smiles>[R1]c1cc(-c2oc3c([R6])c([R3])c([R14])c(O)c3c(=O)c2[R16])cc([R1])c1O</smiles>

(100) $\mathrm{OH}$

(101) $\mathrm{H}$

(102) $\mathrm{OH}$

(103) $\mathrm{OH}$

$\begin{array}{lllll}\mathrm{R} 1 & \mathrm{R} 2 & \mathrm{R} 3 & \mathrm{R} 4 & \mathrm{R}\end{array}$

$\begin{array}{lllllll}\mathrm{H} & \mathrm{OH} & \mathbf{a} & \mathrm{H} & \mathrm{OH} & \mathrm{H}\end{array}$

$\begin{array}{lllllll}\mathrm{H} & \mathrm{H} & \mathrm{H} & \mathbf{e} & \mathrm{OH} & \mathrm{H}\end{array}$

$\begin{array}{lllllllll}\mathrm{H} & \mathrm{H} & \mathrm{H} & \mathrm{H} & \mathrm{OH} & \mathbf{e}\end{array}$

$\begin{array}{lllllll}\mathrm{H} & \mathrm{H} & \mathrm{H} & \mathrm{H} & \mathrm{OH} & \mathrm{H}\end{array}$

$\begin{array}{lllllll}\mathrm{H} & \mathrm{H} & \mathrm{OH} & \mathrm{H} & \mathrm{OH} & \mathrm{H}\end{array}$

$\begin{array}{lllllll}\mathrm{OCH}_{3} & \mathrm{H} & \mathrm{H} & \mathrm{H} & \mathrm{OH} & \mathrm{H}\end{array}$

$\begin{array}{lllllll}\mathrm{H} & \mathrm{OH} & \mathrm{H} & \mathrm{H} & \mathrm{OH} & \mathrm{H}\end{array}$

$\begin{array}{lllllll} & \mathrm{OH} & \mathrm{OH} & \mathrm{H} & \mathrm{OH} & \mathrm{H}\end{array}$

$\begin{array}{lllllll}\mathrm{H} & \mathrm{OH} & \mathrm{OH} & \mathrm{H} & \mathrm{OH} & \mathrm{H}\end{array}$

$\begin{array}{lllllll}\mathrm{OH} & \mathrm{H} & \mathbf{b} & \mathrm{H} & \mathrm{OH} & \mathrm{H}\end{array}$

$\begin{array}{llllllllll}\mathrm{OH} & \mathrm{H} & \mathbf{c} & \mathrm{H} & \mathrm{OH} & \mathrm{H}\end{array}$

$\begin{array}{lllllll}\mathrm{OH} & \mathrm{H} & \mathbf{d} & \mathrm{H} & \mathrm{OH} & \mathrm{H}\end{array}$

(106)<smiles>Cc1c(O)c(C)c2c(c1O)C(=O)CC(c1ccccc1O)O2</smiles>

Figure 5: Structure of Peltogynoids and Flavonoids from genus Bougainvillea. a. Rutinose, b. Rhamnopyranoside, c. RhamnopyranosylRhamnopyranosyl-Galactopyranoside, d. Caffeoyl-rhamnopyranosyl-Rhamnopyranosyl-Galactopyranoside, e. Glucosyl, and f. FeruloylApiofuranosyl-Glucopyranoside.

carrageenin and dextran, as well as arthritis induced with Freund's adjuvant in male Wistar albino rats. The results with methanol extract presented an elevated anti-inflammatory activity for all inflammation models [59].

In the case of ethanol extracts of flowers with bracts from B. $x$ buttiana (var. Orange), the anti-inflammatory properties were measured using the oedema method induced with carrageenin in female CD1 mice. The amounts of cytokines such as IFN- $\gamma$, IL-6, and IL-10 and nitric oxide (NO) were also measured. The results obtained from this extract have shown that it is capable of inducing a decrease in TNF productions and an increment in the IL-6, IFN $\gamma$, IL-10, and NO levels [48]. In another study, the anti-inflammatory effect was obtained in $\mathrm{BALB} / \mathrm{c}$ female mice orally treated with ethanol extract of bracts and flowers from B. x buttiana (var. Rose) [28].

4.3. Antipyretic. The oral administration of methanol extracts from B. glabra in groups of rats showed a significant antipyretic activity [48].

4.4. Antidiabetic. The antidiabetic effects were studied in three species of Bougainvillea. The extracts of leaves from $B$. glabra were used in male Wistar rats induced with alloxan [18]. Similar studies were performed with the oral administration of ethanol extracts of flowers from $B$. spectabilis. Its antidiabetic effect was evaluated using diabetic male Wistar rats induced with alloxan [74]. The chloroform extract of flowers from B. spectabilis administered intraperitoneally reduced glucose levels in diabetic Swiss mice [75]. The antidiabetic effect was also seen with oral administration of aqueous extract from apical leaves of B. spectabilis [61, 76]. Studies carried out on the steam bark extract of $B$. spectabilis orally administered in albino rats showed significant hypoglycaemic activity [77]. In the case of ethanol extracts of bracts and flowers from B. x buttiana, a significant hypoglycaemic activity was observed in female and male CD1 mice orally treated [78].

4.5. Antihyperlipidemic. The treatment of diabetic male Wistar rats induced with alloxan or normal Wistar rats orally treated with different extracts from B. glabra showed the reduction in the amount of total cholesterol (TC), triglycerides (TG), low-density lipoprotein cholesterol (LDL-Cholesterol), and increase high-density lipoprotein 
TABLE 7: Betalains from genus Bougainvillea.

\begin{tabular}{|c|c|c|c|c|}
\hline No. & Compound's name & Species & Parts used & Reference \\
\hline (120) & Bougainvillein-r-I & B. Mrs. Butt & Bracts & [37] \\
\hline (121) & Bougainvillein-r-III & B. Mrs. Butt & Bracts & [37] \\
\hline (122) & 15S-Betanidin 6-O- $\beta$-glucoside & B. glabra & Bracts & [38] \\
\hline (123) & $6^{\prime \prime}$-O-Rhamnosyl-bougainvillein-V & B. glabra & Bracts & [39] \\
\hline (124) & Bougainvillein-V & B. glabra & Bracts & [38] \\
\hline (125) & 15S-Betanidin 6-O(6' $-O$-E-caffeoyl $)$ - $\beta$-sophoroside & B. glabra & Bracts & {$[38]$} \\
\hline (126) & 15S-Betanidin 6-O(6" $-O$-E-4-coumaroyl $)-\beta$-sophoroside & B. glabra & Bracts & {$[38]$} \\
\hline (127) & 15S-Betanidin 6-O(6'-O-E-4-coumaroyl)- $\beta$-sophoroside & B. glabra & Bracts & [38] \\
\hline (128) & $\begin{array}{c}\text { 15S-Betanidin 6-O }\left\{2^{\prime \prime}-O \text { - } \beta \text {-sophorosyl }\left[\left(6^{\prime}-O \text {-E-caffeoyl }\right)-\left(6^{\prime \prime}-O-E-4-\right.\right.\right. \\
\text { coumaroyl })\}-\beta \text {-sophoroside }\end{array}$ & B. glabra & Bracts & [38] \\
\hline (129) & $\begin{aligned} 15 S-B e t a n i d i n ~ 6-O- & \left\{2^{\prime \prime}-O-\beta \text {-glucosyl }\right)\left[6^{\prime}-O \text {-E-caffeoyl }\right)-\left(6^{\prime \prime}-O-E-4-\right. \\
& \text { coumaroyl })]\}-\beta \text {-sophoroside }\end{aligned}$ & B. glabra & Bracts & [38] \\
\hline (130) & 15S-Betanidin 6-O-[2" -O- $\beta$-glucosyl $)\left(6^{\prime}, 6^{\prime \prime}\right.$-di-4-coumaroyl $\left.)\right]-\beta$-sophoroside & B. glabra & Bracts & [38] \\
\hline (131) & 15S-Betanidin 6-O(6', $6^{\prime \prime}$-di-O-E-4-coumaroyl)- $\beta$-sophoroside & B. glabra & Bracts & {$[38]$} \\
\hline$(132)$ & $\begin{array}{c}\text { Betanidin-6-O- }\left[\left(2^{\prime \prime} \text {-O-beta-sophorosyl }\right)-\left(6^{\prime}-\mathrm{O} \text {-trans-feruloyl- } 6^{\prime \prime}-\mathrm{O} \text {-trans- }\right.\right. \\
\text { coumaroyl })] \text {-beta-sophoroside }\end{array}$ & B. glabra & Bracts & [40] \\
\hline (133) & Betanidin-6-O-(6'-O-trans-4-coumaroyl)- $\beta$-sophoroside & B. glabra & Bracts & [40] \\
\hline (134) & $\begin{array}{c}\text { Betanidin-6-O- }\left[\left(2^{\prime \prime}-\mathrm{O}-\beta \text {-sophorosyl }\right)-\left(6^{\prime}, 6^{\prime \prime} \text {-di-O-trans-coumaroyl }\right)\right]-\beta \text { - } \\
\text { sophoroside }\end{array}$ & B. glabra & Bracts & {$[40]$} \\
\hline (135) & $\begin{array}{c}\text { Betanidin-6-O- }\left[\left(2^{\prime \prime}-\mathrm{O}-\beta \text {-glucosyl }\right)-\left(6^{\prime}-\mathrm{O} \text {-trans-caffeoyl- } 6^{\prime \prime}-\mathrm{O}-\right.\right. \\
\text { trans-coumaroyl })]-\beta \text {-sophoroside }\end{array}$ & B. glabra & Bracts & [40] \\
\hline (136) & $\begin{array}{c}\text { Betanidin-6-O- }\left[\left(2^{\prime \prime}-\mathrm{O}-\beta \text {-glucosyl }\right)-\left(6^{\prime}-\mathrm{O} \text {-trans-coumaroyl- } 6^{\prime \prime} \text {-O-trans- }\right.\right. \\
\text { feruloyl })]-\beta \text {-sophoroside }\end{array}$ & B. glabra & Bracts & [40] \\
\hline (137) & $\begin{array}{c}\text { Betanidin-6-O- }\left[(2-\mathrm{O}-\beta \text {-glucosyl })-\left(6^{\prime}, 6^{\prime \prime} \text {-di-O-trans-coumaroyl }\right)\right]-\beta \text { - } \\
\text { sophoroside }\end{array}$ & B. glabra & Bracts & [40] \\
\hline
\end{tabular}

(107)<smiles>CCC(C=CC(C)C1CCC2C3=CCC4CC(O)CCC4(C)C3CCC21C)C(C)C</smiles>

(108)

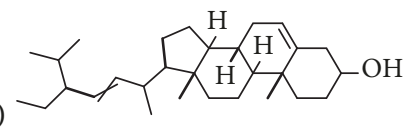

(109)

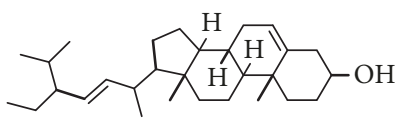

(110)

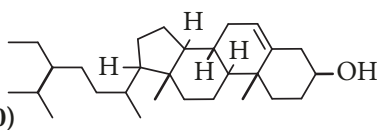

(111)<smiles>CCC(CCC(C)C1CCC2C3=CCC4CC(O)CCC4(C)C3(C)CCC21C)C(C)C</smiles>
(112)

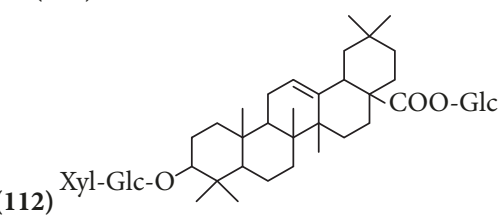

(113)<smiles>CC(C)=CCC/C=C(\C)CCC/C(C)=C/CC/C(C)=C/CC/C=C(\C)CC/C=C(\C)CCC=C(C)C</smiles>
(116)<smiles>CC(C)=CCC/C(C)=C/CC(O)/C=C(\C)CCC=C(C)C</smiles>

(117)<smiles>Cc1c(C)c2c(c(C)c1O)OC(C)(CCCC(C)CCCC(C)CCCC(C)I)CC2</smiles>

(118)

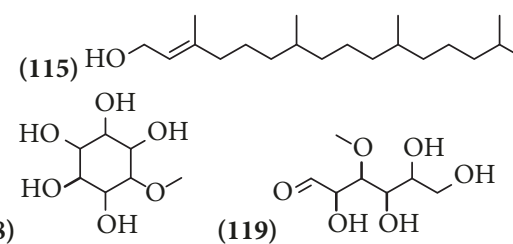

FIGURE 6: Structure of phytosterols, terpenes, and carbohydrates from genus Bougainvillea.

cholesterol (HDL-C) [18]. Another study, using Wistar rats exposed to oral injection of ethanol extract of fresh leaves from B. spectabilis, showed a significant reduction in total cholesterol (TC), triglyceride (TG), low-density lipoprotein (LDL), and very low-density lipoprotein (VLDL) levels and significant $(p<0.01)$ increase in high-density lipoproteins (HDL) in hypercholesterolemia rats [20].
4.6. Antidiarrhoeal. A significant antidiarrhoeal activity was observed in male Wistar rats orally treated with the acetone extract obtained from leaves of B. glabra "Choicy" [45].

4.7. Antiulcer. The oral administration of extracts of acetone of leaves of B. glabra "Choicy" and their antiulcer effect were 

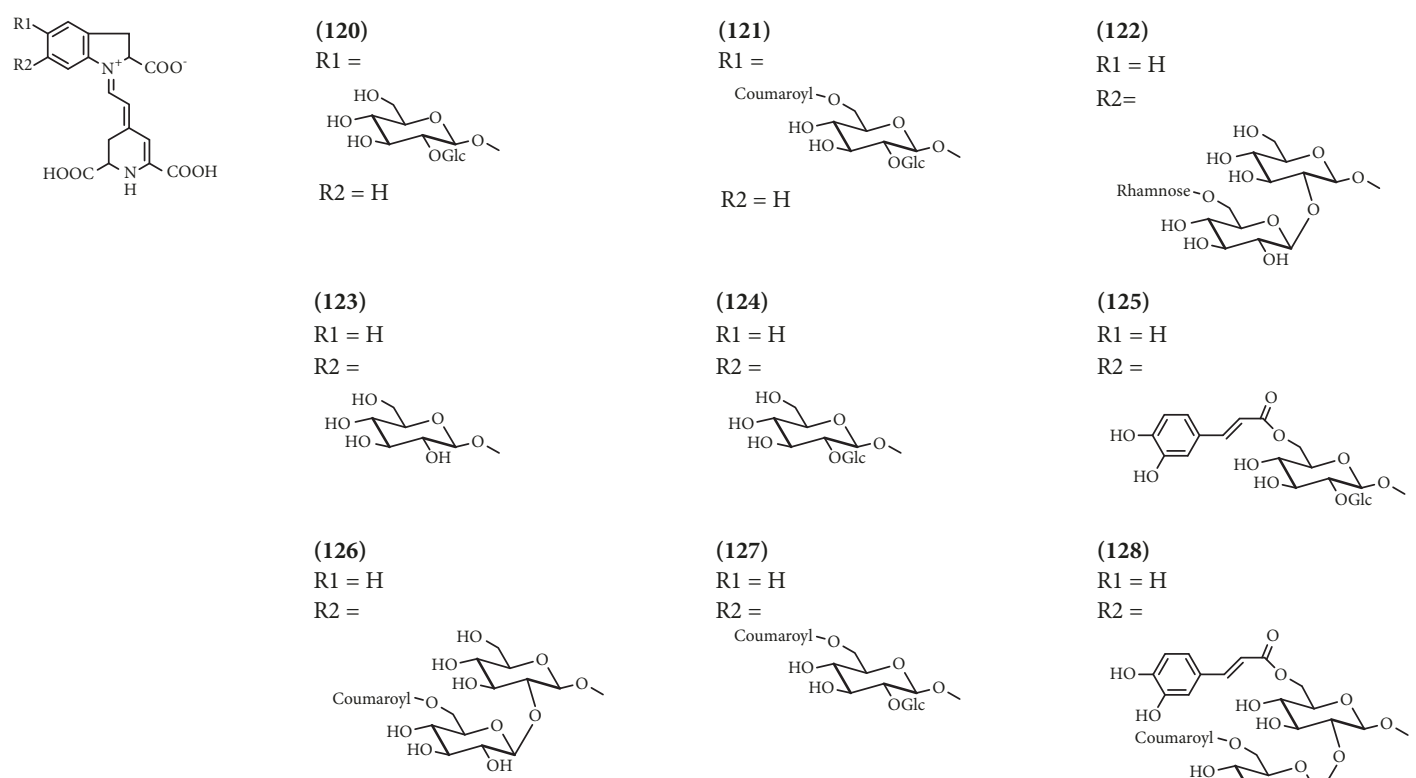

(128)
$\mathrm{R} 1=\mathrm{H}$
$\mathrm{R} 2=$

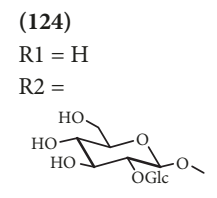

$\mathrm{R} 1=\mathrm{H}$

$\mathrm{R} 2=$
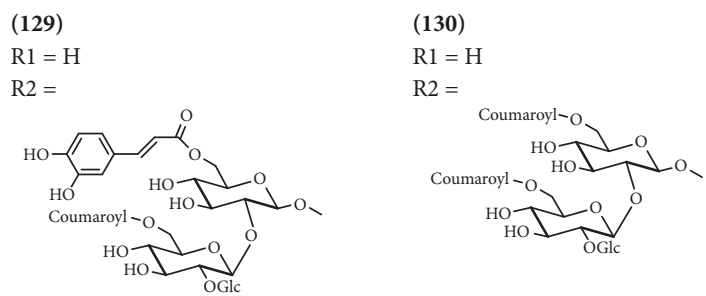

(131)

$\mathrm{R} 1=\mathrm{H}$

$\mathrm{R} 2=$
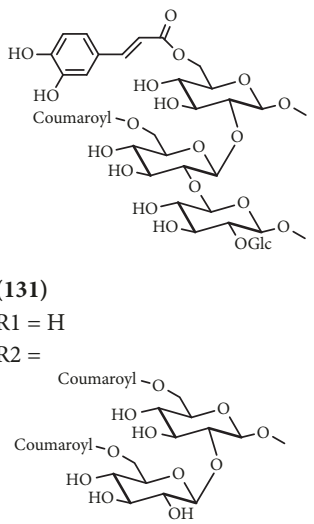

(132)

$\mathrm{R} 1=\mathrm{H}$

(133)

$\mathrm{R} 1=\mathrm{H}$

$\mathrm{R} 2=\mathrm{OH}$

R2

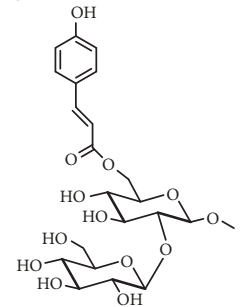

(134)

$\mathrm{R} 1=\mathrm{H}$
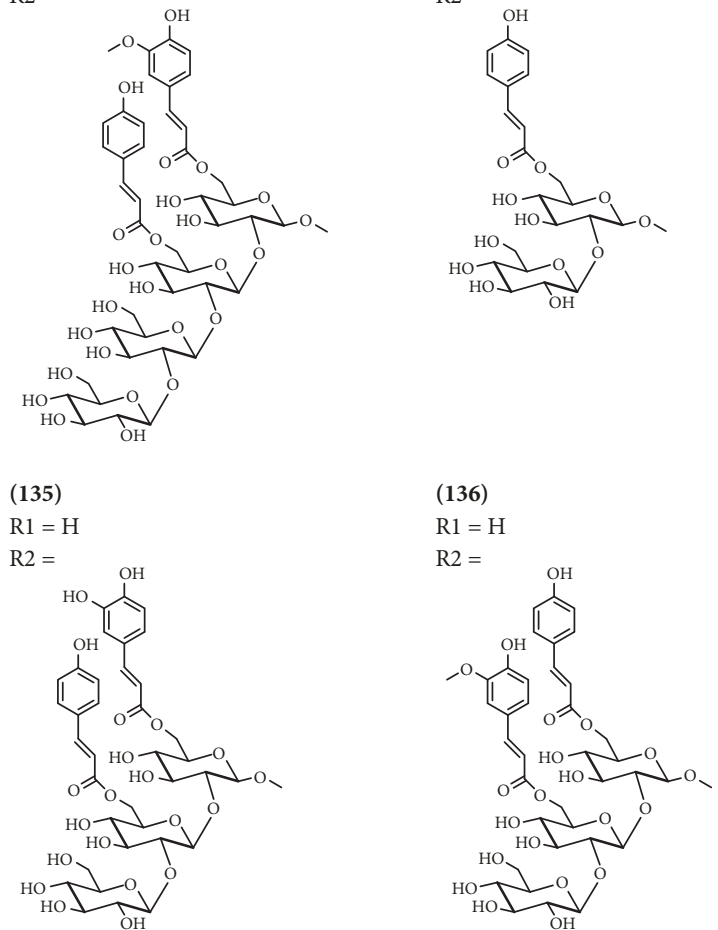

(137)

$\mathrm{R} 1=\mathrm{H}$

$\mathrm{R} 2=$

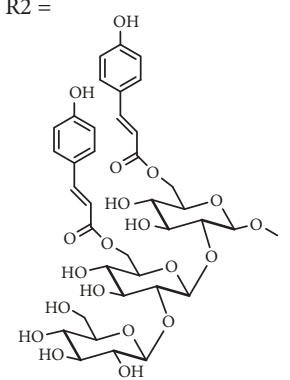

FIGURE 7: Structure of betalains from genus Bougainvillea. 
evaluated in male Wistar rats, and this extract showed a marked antiulcer activity [45].

4.8. Antifertility. A reduction in testosterone and oestrogen levels [21] as well as sperm count, viability, and motility [56] was observed in albino Swiss male and female mice orally treated with ethanol extract from B. spectabilis.

4.9. Neuroprotective. The neuroprotective effect of leaves from B. glabra extracted with ethanol was evaluated by the use of the mortality of Drosophila melanogaster flies. The results obtained showed that the flies treated with the extract present a lower mortality [32].

The effect of two methanol extracts of B. spectabilis from yellow and pink bracts on oxidative stress and neural damage was carried out by use of male Sprague-Dawley rats subcutaneously injected with rotenone. Rotenone provoked significant increases of brain MDA (product of lipid peroxidation) and nitric oxide content along with decreased brain reduced glutathione. There was also a marked and significant inhibition of brain paraoxonase-1 (PON-1) and butyrylcholinesterase (BChE) activities and increased proinflammatory cytokine interleukin-lbeta (Il-1 $\beta)$ in brain of rotenone-treated rats. B. spectabilis flowers extract itself resulted in increased brain oxidative stress, lipid peroxidation, and nitrite content while inhibiting PON-1 activity. The yellow flowers extract inhibited BChE activity and increased brain Il-1 $\beta$. When given to rotenone-treated rats, $B$. spectabilis extracts, however, decreased lipid peroxidation while their low administered doses increased brain glutathione (GSH). Brain nitrite decreased with the pink extract but showed further increase with the yellow extract. Both extracts caused further inhibition of PON-1 activity while the yellow extract resulted in further inhibition of BChE activity. Histopathological studies indicated that both extracts protected against brain, liver, and kidney damage caused by the toxicant [52].

4.10. Thrombolytic. The methanol extract of leaves from $B$. glabra [53] and an aqueous extract of green leaves from $B$. spectabilis [54] showed the thrombolytic activity in vitro in the blood of healthy volunteers.

4.11. Cardiotonic. Cardiotonic evaluation of an aqueous extract of B. glabra was performed by using isolated frog heart perfusion technique. The parameters studied included contraction force (HR), heart rate (HR), and cardiac output (CO). This extract provoked an increase in HR and CO [71].

4.12. Anthelmintic. For the anthelmintic evaluation, methanol extracts from B. glabra were used against species of Pheretima posthuma [62], and ethanol Eudrilus eugeniae and Eisenia foetida [51] were compared with the standards albendazole and metronidazole, respectively. All extracts of B. glabra could cause paralysis and death of worms $[51,62]$.

4.13. Antimicrobial. The conventional methods used for the evaluation of biological properties such as antibacterial and antifungal agents of plant extracts include the agar diffusion method and the dilution method $[60,79]$ (Table 8).
4.14. Plant Antiviral. The leaf proteins from B. x buttiana were evaluated against RNA viruses such as tobamoviruses, tobacco mosaic virus, and sunnhemp rosette virus. The results obtained showed a degradation of viral RNAs. This implies a great opportunity for control of vegetable viruses [69]. In another study, this purification could identify lysine as the inhibitor of N-glycosidase activity on the 25S rRNA ribosomes of tobacco by interfering with viral multiplication [70].

4.15. Cytotoxic. The cytotoxic effect of ethanol extract of leaves from B. glabra was evaluated in HT-29 cells, AGS, and BL-13 [16]. Another study with stems and leaves from $B$. glabra extracted with acetonitrile, butanol, dichloromethane, ethyl acetate, hexane, and methanol showed the antiproliferative activity against $\mathrm{U} 373$ cells [72].

The effect of the antiproliferative activity in U373 cells was evaluated using extracts hexane, dichloromethane, acetonitrile, ethyl acetate, methanol, and butanol extracts from stems and leaves from B. spectabilis. The extract of dichloromethane showed lower antiproliferative activity when compared to others extracts [72]. In another study, the cytotoxic activity of eight new compounds named bougainvinones 7885 isolated and elucidated from stem bark was evaluated. The extract from B. spectabilis purple from a bipartition was evaluated by using KB, HeLa S-3, HT-29, MCF-7, and HepG2. The results showed that the compound $\mathbf{8 4}$ showed cytotoxicity against cancer cell lines and compounds 79 and 80 exhibited cytotoxicity against the KB cell line [24]. In a subsequent study, in ethylacetate extract, five new flavones named bougainvinones 86-90 were isolated and elucidated and their cytotoxic activities were assayed against KB, HepG2, HeLa, S-3, HT-29, and MCF cells. The results showed that all compounds had cytotoxic activity [33].

The cytotoxic effect of flower from B. x buttiana extracted in ethanol in bracts of different colours, orange-1 (Bxb01), orange-2 (Bxb02), pink (BxbR), violet (BxbV), and white (BxbW), was studied on HeLa cells. The greater cytotoxic activity was observed with the bracts with flowers Bxb02 and Bxb01 [63]. In another study the evaluation of the cytotoxic activity of different extracts, aqueous, methanol, ethanol, acetone, ethylacetate, dichloromethane, and hexane, of bracts with flowers from B. $x$ buttiana was performed. The dichloromethane extract was the most cytotoxic to L-929 cells [26].

4.16. Immunomodulatory. The effect of an ethanol extract from $B$. x buttiana on the activation of macrophages of female $\mathrm{CD} 1$ mice was determined. The results obtained showed an increase in $\mathrm{H}_{2} \mathrm{O}_{2}$ levels and the extension and formation of vacuoles, reduction of TNF- $\alpha$, and remarkable increases for the levels of IL-10 and NO, suggesting an immunomodulatory effect [55].

4.17. Antioxidants. The fresh leaves were degreased with petroleum ether followed by a successive extraction of $B$. buttiana with acetone, ethanol, and distilled water. They were then used for the determination of the antioxidant capacity using the methods of DPPH, FRAP, and inhibition of lipid 
TABLE 8: Antibacterial and antifungal activities found in genus Bougainvillea.

\begin{tabular}{|c|c|c|c|}
\hline Microorganism & Species & Extract & Reference \\
\hline \multirow[t]{6}{*}{ Bacillus subtilis } & B. spectabilis & Methanol & {$[23]$} \\
\hline & B. spectabilis & Ethanol, water, chloroform, and ethyl acetate & [41] \\
\hline & B. spectabilis & Chloroform partition & {$[42]$} \\
\hline & Snow White & Ethanol & [43] \\
\hline & B. glabra & Methanol & {$[11,44]$} \\
\hline & B. glabra "Choicy" & Acetone & [45] \\
\hline Bacillus megaterium & B. spectabilis & Chloroform partition & {$[42]$} \\
\hline Bacillus cereus & B. spectabilis & Chloroform partition & {$[42]$} \\
\hline \multirow[t]{6}{*}{ Escherichia coli } & B. spectabilis & Methanol & {$[23]$} \\
\hline & B. spectabilis & Chloroform partition & {$[42]$} \\
\hline & B. spectabilis & Acetone, chloroform, methanol, petroleum ether, and water & {$[46]$} \\
\hline & B. glabra "Choicy" & Acetone & {$[45]$} \\
\hline & Snow White & Ethanol & {$[43]$} \\
\hline & B. glabra & Methanol & {$[11,44]$} \\
\hline Shigella flexneri & B. spectabilis & Methanol & {$[23]$} \\
\hline Shigella boydii & B. spectabilis & Chloroform partition & {$[42]$} \\
\hline Shigella dysenteriae & B. spectabilis & Chloroform partition & {$[42]$} \\
\hline Sarcina lutea & B. spectabilis & Chloroform partition & {$[42]$} \\
\hline \multirow[t]{4}{*}{ Klebsiella pneumoniae } & B. spectabilis & Aqueous & {$[41]$} \\
\hline & B. spectabilis & Acetone, chloroform, methanol, petroleum ether, and water & {$[46]$} \\
\hline & B. glabra "Choicy" & Acetone & {$[45]$} \\
\hline & Snow White & Ethanol & {$[43]$} \\
\hline \multirow[t]{4}{*}{ Staphylococcus aureus } & B. spectabilis & Chloroform partition & {$[42]$} \\
\hline & B. spectabilis & Petroleum ether and chloroform & {$[46]$} \\
\hline & B. glabra "Choicy" & Acetone & {$[45]$} \\
\hline & Snow White & Ethanol & {$[43]$} \\
\hline \multirow[t]{3}{*}{ Proteus vulgaris } & B. spectabilis & Ethanol and chloroform & {$[41]$} \\
\hline & B. glabra "Choicy" & Acetone & {$[45]$} \\
\hline & Snow White & Ethanol & {$[43]$} \\
\hline \multirow[t]{3}{*}{ Salmonella typhi } & B. spectabilis & Methanol & {$[23]$} \\
\hline & B. spectabilis & Chloroform partition & {$[42]$} \\
\hline & Snow White & Ethanol & {$[43]$} \\
\hline Salmonella paratyphi & B. spectabilis & Chloroform partition & {$[42]$} \\
\hline \multirow[t]{2}{*}{ Vibrio cholerae } & B. spectabilis & Acetone, chloroform, methanol, and petroleum ether & {$[46]$} \\
\hline & Snow White & Ethanol & {$[43]$} \\
\hline Vibrio mimicus & B. spectabilis & Chloroform partition & {$[42]$} \\
\hline Vibrio parahaemolyticus & B. spectabilis & Chloroform partition & {$[42]$} \\
\hline \multirow[t]{3}{*}{ Pseudomonas aeruginosa } & B. spectabilis & Methanol & {$[23]$} \\
\hline & B. spectabilis & Chloroform partition & {$[42]$} \\
\hline & B. glabra & Methanol & {$[11,44]$} \\
\hline \multirow[t]{2}{*}{ Candida albicans } & B. spectabilis & Chloroform partition & {$[42]$} \\
\hline & B. glabra & Methanol & [47] \\
\hline \multirow[t]{2}{*}{ Aspergillus fumigatus } & B. spectabilis & Acetone, chloroform, methanol, petroleum ether, and water & {$[46]$} \\
\hline & B. glabra & Methanol & [47] \\
\hline Aspergillus flavus & B. spectabilis & Acetone, chloroform, methanol, petroleum ether, and water & {$[46]$} \\
\hline \multirow[t]{3}{*}{ Aspergillus niger } & B. spectabilis & Ethanol, water, and chloroform & [41] \\
\hline & B. spectabilis & Chloroform partition & {$[42]$} \\
\hline & B. spectabilis & Acetone, chloroform, methanol, petroleum ether, and water & [46] \\
\hline Trichoderma viridae & B. spectabilis & Ethanol and chloroform & {$[41]$} \\
\hline Penicillium notatum & B. spectabilis & Ethanol & {$[41]$} \\
\hline Rhizopus oryzae & B. spectabilis & Ethyl acetate & {$[41]$} \\
\hline Coccidioides immitis & B. glabra & Methanol & [47] \\
\hline
\end{tabular}


TABLE 9: Summarized activities found in genus Bougainvillea.

\begin{tabular}{|c|c|c|c|c|c|c|}
\hline \multirow{2}{*}{ Activity } & \multicolumn{5}{|c|}{ Species } & \multirow{2}{*}{ References } \\
\hline & B. buttiana & B. glabra & B. spectabilis & B.peruviana & B. $x$ buttiana & \\
\hline Analgesic & & $\sqrt{ }$ & & & $\sqrt{ }$ & {$[29,48,49]$} \\
\hline Anthelmintic & & $\sqrt{ }$ & & & & {$[50,51]$} \\
\hline Antidiabetic & & $\sqrt{ }$ & $\sqrt{ }$ & & $\sqrt{ }$ & {$[16,42,52-55]$} \\
\hline Antidiarrheal & & $\sqrt{ }$ & & & & {$[45]$} \\
\hline Antifertility & & & $\sqrt{ }$ & & & {$[21,56]$} \\
\hline Antihyperlipidemic & & $\sqrt{ }$ & $\sqrt{ }$ & & & {$[18,29,57,58]$} \\
\hline Anti-inflammatory & & $\sqrt{ }$ & $\sqrt{ }$ & & $\sqrt{ }$ & {$[28,48-50,59]$} \\
\hline Antimicrobial & & $\sqrt{ }$ & $\sqrt{ }$ & & & {$[11,23,41-47,60]$} \\
\hline Antioxidants & $\sqrt{ }$ & $\sqrt{ }$ & $\sqrt{ }$ & $\sqrt{ }$ & $\sqrt{ }$ & {$[11,16,26,46,61-68]$} \\
\hline Antipyretic & & $\sqrt{ }$ & & & & {$[48]$} \\
\hline Antiulcer & & $\sqrt{ }$ & & & & {$[45]$} \\
\hline Antiviral & & & & & $\sqrt{ }$ & {$[69,70]$} \\
\hline Cardiotonic & & $\sqrt{ }$ & & & & {$[71]$} \\
\hline Cytotoxic & & $\sqrt{ }$ & & & $\sqrt{ }$ & {$[16,24,26,33,63,72]$} \\
\hline Immunomodulator & & & & & $\sqrt{ }$ & {$[55]$} \\
\hline Neuroprotective & & $\sqrt{ }$ & $\sqrt{ }$ & & & {$[32,52]$} \\
\hline Thrombolytic & & $\sqrt{ }$ & $\sqrt{ }$ & & & {$[53,54]$} \\
\hline
\end{tabular}

Note: the symbol $\sqrt{ }$ represents the present biological or pharmacological activity in the respective species.

peroxidation. The ethanol extracts of B. buttiana showed antioxidant activity using the two methods studied and an inhibitory activity of lipid peroxidation $[63,64]$ was observed.

Another study was performed to determine the antioxidant activity of four flowers of a population of Thailand, including B. glabra. The extraction was carried out using acidified ethanol and the antioxidant activity was detected using the FRAP and ORAC methods [16]. In another study, the antioxidant activities of different extracts of hexane, dichloromethane, acetonitrile, ethyl acetate, methanol, and butanol were evaluated using the DPPH, ABTS, FRAP, and lipid peroxidation methods. The butanol and methanol extracts showed high antioxidant activity by all methods used [72]. B. glabra bracts extracted from methanol also showed high levels of NO and antioxidant activity [44, 6567]. Antioxidant activity was also detected in bracts with flowers extracted in methanol and subsequently partitioned with hexane, chloroform, and water [11].

Fresh leaves from $B$. peruviana were first degreased with petroleum ether and were subsequent to extraction with acetone, distilled water, and ethanol and their antioxidant activity was evaluated by the methods of DPPH and lipid peroxidation inhibition. In ethanolic extracts from $B$. peruviana the antioxidant activity was also detected [64].

Fresh leaves from B. spectabilis were extracted with methanol and water. The results showed antioxidant activity in both extracts [68]. The use of apical leaves from B. spectabilis extracted with distilled water measured the biomarkers of oxidative stress in blood in diabetic male Wistar rats induced with streptozotocin. The results showed that diabetic rats presented a significant decrease in GSH, SOD, and catalase [61]. Another study of B. spectabilis leaves extracted with acetone, chloroform, methanol, petroleum ether, and water also showed the high antioxidant activity [46].
The antioxidant activity of an ethanol extract of bracts of different colours from B. $x$ buttiana was determined using the DPPH method. All extracts presented antioxidant activity, and the percent of radical scavenging activity was dependent on the colour of bracts [46]. In another study, the extracts from B. $x$ buttiana with water, methanol, ethanol, acetone, ethylacetate, dichloromethane, and hexane were used to evaluate their antioxidant activity using the DPPH method. The percent radical scavenging activities in order were methanol $>$ ethanol $>$ water $>$ acetone $>$ ethylacetate $>$ hexane $>$ dichloromethane [26]. Table 9 summarizes the activities found in the genus Bougainvillea.

\section{Toxicity}

The leaves of B. glabra "Choicy" were subjected to successive extractions with different solvents such as acetone, ethanol, and water. This extract was orally administered in albino rats of both sexes to assess toxicity. The results obtained did not show death in these animals [45].

The evaluation of the toxic effect of aqueous extracts of leaves from $B$. spectabilis was carried out in mice intragastrically treated [7]. Leaves from $B$. spectabilis extracted with ethanol were used in albino male rats (Rattus norvegicus) to evaluate the haemoglobin concentration $(\mathrm{Hb})$, packed cell volume (PVC), red blood cell count (RBC), mean corpuscular haemoglobin concentration (MCHC), mean corpuscular volume (MCV), white blood cell count (WBC), and platelet count (PLC). The extract orally administered provoked a significant reduction of $\mathrm{Hb}, \mathrm{RBC}$, and PCV [20]. Another investigation with aqueous extracts of leaves from B. spectabilis orally administered in male and female albino Swiss mice showed a significant reduction in haemoglobin, 
red blood cell, and haematocrit levels. This may also be a cause of anemia [80]. The acute toxicity was also evaluated in Wistar albino rats treated with ethanol extracts of root bark. The results showed no toxic effects in the rats [74]. However, the methanol extract from B. spectabilis after oral treatment was toxic in male Wistar rats [50]. Other studies showed that the oral administration of methanol extract from B. spectabilis in male Swiss mice not was causative of renal or hepatic damage [59].

The toxicity effect of flowers of different colours (white, orange, shocking pink, red, and violet) from B. spectabilis extracted with methanol was evaluated on brine shrimp Artemia salina [23]. The results obtained showed no toxicity effect presented on Artemia [75].

The toxicity effect of ethanol extracts of bracts from $B . x$ buttiana was carried out on female CD1 and/or BALB/c mice. The results obtained showed that these extracts were not toxic for two different strains of mice [28].

\section{Conclusions}

This review details the ethnomedical, phytochemical, and pharmacological and toxicological uses of the different species, cultivars, and hybrids of Bougainvillea. Although there are several studies on the pharmacological activity of the genus Bougainvillea, there is potential of this plant as an anti-inflammatory, antioxidant, immunomodulator, antimicrobial, etc.

\section{Disclosure}

The role of the funding sponsors was with the scholarship payment and had no role in the study design, in the collection, analysis, or interpretation of data, in the writing of the manuscript, or in the decision to publish the results.

\section{Conflicts of Interest}

The authors declare no conflicts of interest.

\section{Authors' Contributions}

Rodolfo Abarca-Vargas and Vera L. Petricevich contributed to this work, prepared the manuscript, and approved this version of the article.

\section{Acknowledgments}

This work was supported by Secretaría de Educación Publica (SEP-PROMEP) and Consejo Nacional de Ciencia y Tecnología (CONACyT), Mexico.

\section{References}

[1] The Plants List. Bougainvillea. Available at: http://www.theplantlist.org./tpl1.1/search?q=Bougainvillea. (Accessed: 1st October 2017).
[2] K. D. Kobayashi, J. McConnell, and J. Griffis, Bougainvillea. Ornamentals and Flowersk, Available at: http://scholarspace .manoa.hawaii.edu/bitstream/10125/2959/1/OF-38.pdf., 2007.

[3] R. Gobato, A. Gobato, and D. F. G. Fedrigo, "Study of the molecular electrostatic potential of D-Pinitol an active hypoglycemic principle found in Spring flower-Three Marys, (Bougainvillea species) in the Mm+ method," Parana Journal of Science and Education, vol. 2, pp. 1-9, 2016.

[4] N. Sahu and J. Saxena, "Bougainvillea glabra a Natural Antioxidant: A Review," Chemistry, vol. 46, pp. 4113-4117, 2012.

[5] C. Monroy-Ortiz and R. Monroy, "Las plantas, compañeras de siempre: la experiencia en Morelos," (UAEM, Centro de Investigaciones Biológicas de la CONABIO CONANP), 2006.

[6] S. Tripathi, S. Singh, and R. K. Roy, "Pollen morphology of Bougainvillea (Nyctaginaceae): A popular ornamental plant of tropical and sub-tropical gardens of the world," Review of Palaeobotany and Palynology, vol. 239, pp. 31-46, 2017.

[7] A. Argueta Villamar, L. M. Cano Asseleih, and M. L. Rodarte, Atlas de las plantas de las Medicina Tradicional Mexicana, Instituto Nacional Indigenista, 1994.

[8] S. Sangthong, P. Suksabye, and P. Thiravetyan, "Air-borne xylene degradation by Bougainvillea buttiana and the role of epiphytic bacteria in the degradation," Ecotoxicology and Environmental Safety, vol. 126, pp. 273-280, 2016.

[9] M. Piattelli and F. Imperato, "Pigments of Bougainvillea glabra," Phytochemistry, vol. 9, no. 12, pp. 2557-2560, 1970.

[10] A. Beltrà, A. Soto, J. Germain et al., "The Bougainvillea mealybug Phenacoccus peruvianus, a rapid invader from South America to Europe," Entomologia Hellenica, vol. 19, no. 2, p. 137, 2017.

[11] M. Z. Islam, M. T. Hossain, F. Hossen, M. S. Akter, and M. A. Mokammel, "In-vitro antioxidant and antimicrobial activity of Bougainvillea glabra flower," Research Journal of Medicinal Plant, vol. 10, no. 3, pp. 228-236, 2016.

[12] J. P. Maran, B. Priya, and C. V. Nivetha, "Optimization of ultrasound-assisted extraction of natural pigments from Bougainvillea glabra flowers," Industrial Crops and Products, vol. 63, pp. 182-189, 2015.

[13] J. Yang, K. Zhang, D. Li et al., "Morphological investigation and classification research of 18 bougainvillea," in Proceedings of the 8th International Conference on Measuring Technology and Mechatronics Automation, ICMTMA '16, pp. 401-404, March 2016.

[14] A. H. Ahmed, "New flavone from the aerial parts of Bougainvillea glabra," International Journal of Computer Engineering Research, vol. 4, pp. 1-5, 2014.

[15] Y. Schlein, R. L. Jacobson, and G. C. Müller, "Sand fly feeding on noxious plants: A potential method for the control of Leishmaniasis," The American Journal of Tropical Medicine and Hygiene, vol. 65, no. 4, pp. 300-303, 2001.

[16] O. Kaisoon, I. Konczak, and S. Siriamornpun, "Potential health enhancing properties of edible flowers from Thailand," Food Research International, vol. 46, no. 2, pp. 563-571, 2012.

[17] Y. J. Perales and M. Leysa, "Phytochemical screening and antibacterial acitivity of Bougainvillea glabra plant extract as potential sources of antibacterial and resistance-modifying agents," in Proceedings of the International Conference on Life Science and Engineering, pp. 121-125, 2012.

[18] G. I. Adebayo, O. T. Alabi, B. V. Owoyele, and A. O. Soladoye, "Anti-diabetic properties of the aqueous leaf extract of Bougainvillea glabra (Glory of the Garden) on alloxan-induced 
diabetic rats," Records of Natural Products, vol. 3, no. 4, pp. 187192, 2009.

[19] R. Srivastava, S. Shukla, A. Soni, and A. Kumar, "RAPD-based genetic relationships in different Bougainvillea cultivars," Crop Breeding and Applied Biotechnology, vol. 9, no. 2, pp. 154-163, 2009.

[20] J. O. Adebayo, A. A. Adesokan, L. A. Olatunji, D. O. Buoro, and A. O. Soladoye, "Effect of ethanolic extract of Bougainvillea spectabilis leaves on haematological and serum lipid variables in rats," Biokemistri, vol. 17, no. 1, pp. 45-50, 2005.

[21] N. Mishra, S. Joshi, V. L. Tandon, and A. Munjal, "Evaluation of anti-fertility potential of aqueous extract of bougainvillea spectabilis leaves in swiss albino mice," International Journal of Pharmaceutical Sciences and Drug Research, vol. 1, pp. 19-23, 2009.

[22] N. Vukovic, M. Kacaniova, L. Hleba, and S. Sukdolak, "Chemical Composition of the Essential oil of Bougainvillea spectabilis from Montenegro," Journal of Essential Oil Bearing Plants, vol. 16, no. 2, pp. 212-215, 2013.

[23] M. S. Ali, S. A. Ibrahim, F. Ahmed, and M. K. Pervez, "Color versus bioactivity in the flowers of Bougainvillea spectabilis (Nyctaginaceae)," Natural Product Research (Formerly Natural Product Letters), vol. 19, no. 1, pp. 1-5, 2005.

[24] L. T. M. Do, T. Aree, P. Siripong, T. N. K. Pham, P. K. P. Nguyen, and S. Tip-Pyang, "Bougainvinones A-H, peltogynoids from the stem bark of purple bougainvillea spectabilis and their cytotoxic activity," Journal of Natural Products, vol. 79, no. 4, pp. 939-945, 2016.

[25] A. J. Bisigato, M. V. Campanella, and G. E. Pazos, "Plant phenology as affected by land degradation in the arid Patagonian Monte, Argentina: A multivariate approach," Journal of Arid Environments, vol. 91, pp. 79-87, 2013.

[26] R. Abarca-Vargas, C. F. Peña Malacara, and V. L. Petricevich, "Characterization of chemical compounds with antioxidant and cytotoxic activities in bougainvillea $x$ buttiana holttum and standl, (Var. rose) extracts," Antioxidants, vol. 5, no. 4, article no. 45, 2016.

[27] A. J. Alonso-Castro, J. R. Zapata-Morales, A. J. Ruiz-Padilla et al., "Use of medicinal plants by health professionals in Mexico," Journal of Ethnopharmacology, vol. 198, pp. 81-86, 2017.

[28] R. V. Guerrero, R. A. Vargas, and V. L. Petricevich, "Chemical compounds and biological activity of an extract from bougainvillea x buttiana (var. rose) holttum and standl," International Journal of Pharmacy and Pharmaceutical Sciences, vol. 9, no. 3, pp. 42-46, 2017.

[29] R. V. Guerrero, R. Abarca-Vargas, and V. L. Petricevich, "Analgesic effect of ethanolic extract of Bougainvillea x buttiana (Var. Rose) Holttum Standl," EC Pharmacol. Toxicol, vol. 2, pp. 224230, 2016.

[30] L. Arteaga Figueroa, R. Abarca-Vargas, C. García Alanis, and V. L. Petricevich, "Comparison between peritoneal macrophage activation by Bougainvillea $x$ buttiana extract and LPS and/or interleukins," BioMed Research International, vol. 2017, 11 pages, 2017.

[31] S. Singh, S. K. Reddu, S. K. Sharma, and M. Ali, "New unsaturated fatty acid from roots of Bougainvillea spectabilis Willd," Asian Journal of Chemistry, vol. 21, no. 6, pp. 4744-4748, 2009.

[32] J. J. Soares, D. T. Rodrigues, M. B. Gonçalves et al., "Paraquat exposure-induced Parkinson's disease-like symptoms and oxidative stress in Drosophila melanogaster: Neuroprotective effect of Bougainvillea glabra Choisy," Biomedicine \& Pharmacotherapy, vol. 95, pp. 245-251, 2017.

[33] L. T. Do, T. Aree, P. Siripong et al., "Cytotoxic flavones from the stem bark of bougainvillea spectabilis willd," Planta Medica, vol. 84, no. 02, pp. 129-134, 2018.

[34] S. Jawla, Y. Kumar, and M. S. Y. Khan, "Isolation of antidiabetic principle from Bougainvillea spectabilis willd (Nyctaginaceae) stem bark," Tropical Journal of Pharmaceutical Research, vol. 12, no. 5, pp. 761-765, 2013.

[35] A. Simon, G. Tóth, H. Duddeck, H. S. M. Soliman, I. I. Mahmoud, and H. Samir, "Glycosides from Bougainvillea glabra," Natural Product Research (Formerly Natural Product Letters), vol. 20, no. 1, pp. 63-67, 2006.

[36] C. R. Nayayanan, D. D. Joshi, A. M. Mujumdar, and V. V. Dhekne, "Pinitol-A new anti-diabetic compound from the leaves of Bougainvillea spectabilis," Current Science, vol. 56, pp. 139-141, 1987.

[37] M. Piattelli and F. Imperato, "Betacyanins from bougainvillea," Phytochemistry, vol. 9, no. 2, pp. 455-458, 1970.

[38] S. Heuer, S. Richter, J. W. Metzger, V. Wray, M. Nimtzt, and D. Strack, "Betacyanins from bracts of Bougainvillea glabra," Phytochemistry, vol. 37, no. 3, pp. 761-767, 1994.

[39] F. A. Imperato, "A branched trisaccharide in the betacyanins of Bougainvillea glabra," Phytochemistry, vol. 14, no. 11, p. 2526, 1975.

[40] G. Jerz, S. Wybraniec, N. Gebers, and P. Winterhalter, “Targetguided separation of Bougainvillea glabra betacyanins by direct coupling of preparative ion-pair high-speed countercurrent chromatography and electrospray ionization massspectrometry," Journal of Chromatography A, vol. 1217, no. 27, pp. 4544-4554, 2010.

[41] M. Kumara Swamy, K. M. Sudipta, P. Lokesh et al., "Phytochemical screening and in vitro antimicrobial activity of Bougainvillea spectabilis flower extracts," International Journal of Phytomedicine, vol. 4, no. 3, pp. 375-379, 2012.

[42] F. Chowdhury, S. Pal, T. Sharmin et al., "Bioactivities of artocarpus chaplasha roxb. and bougainvillea spectabillis willd," Bangladesh Pharmaceutical Journal, vol. 16, pp. 63-68, 2013.

[43] V. Gupta, M. George, L. Joseph, M. Singhal, and H. P. Singh, "Evaluation of antibacterial activity of Bougainvillea glabra 'snow white' and Bougainvillea glabra 'choicy"' Journal of Chemical and Pharmaceutical Research, vol. 1, pp. 233-237, 2009.

[44] A. Napoleon, S. K. Swetha, and G. Angajala, "In-vitro antioxidant and antibacterial studies of betacyanin isolated from the bracts of Bougainvillea glabra," International Journal of Pharmacy and Pharmaceutical Sciences, vol. 5, pp. 84-87, 2013.

[45] E. Edwin, E. Sheeja, E. Toppo, V. Tiwari, and K. R. Dutt, "Efecto antimicrobiano, antiulceroso y antidiarreico de las hojas de buganvilla (Bougainvillea glabra Choisy)," Ars Pharmaceutica, vol. 48, pp. 135-144, 2007.

[46] S. Dhankhar, M. Sharma, S. Ruhil, M. Balhara, M. Kumar, and A. K. Chhillar, "Evaluation of antimicrobial and antioxidant activities of Bougainvillea spectabilis," International Journal of Pharmacy and Pharmaceutical Sciences, vol. 5, no. 3, pp. 178182, 2013.

[47] B. A. Alanís-Garza, G. M. González-González, R. SalazarAranda, N. Waksman de Torres, and V. M. Rivas-Galindo, "Screening of antifungal activity of plants from the northeast of Mexico," Journal of Ethnopharmacology, vol. 114, no. 3, pp. 468471, 2007. 
[48] A. Elumalai, M. C. Eswaraiah, K. M. Lahari, and H. A. Shaik, "In-vivo screening of Bougainvillea glabra leaves for its analgesic, antipyretic and anti-inflammatory activities," Asian Journal of Pharmaceutical Sciences, vol. 2, pp. 85-87, 2012.

[49] A. L. Alvarez Perez Gil, L. Barbosa Navarro, M. Patipo Vera, and V. L. Petricevich, "Anti-inflammatory and antinociceptive activities of the ethanolic extract of Bougainvillea $x$ buttiana," Journal of Ethnopharmacology, vol. 144, no. 3, pp. 712-719, 2012.

[50] E. Manivannan, R. Kothai, B. Arul, and S. Rajaram, "Antiinflammatory activity of bougainvillea spectabilis linn," Research Journal of Pharmaceutical, Biological and Chemical Sciences, vol. 3, no. 1, pp. 642-646, 2012.

[51] M. C. Eswaraiah, A. Elumalai, A. Boddupalli, and R. K. Gollapalli, "Evaluation of anthelmintic activity of Bougainvillea glabra leaves," Journal of Natural Products, vol. 21, pp. 16-19, 2012.

[52] O. M. E. Abdel-Salam, E. R. Youness, N. A. Ahmed et al., "Bougainvillea spectabilis flowers extract protects against the rotenone-induced toxicity," Asian Pacific Journal of Tropical Medicine, vol. 10, no. 5, pp. 478-490, 2017.

[53] A. Elumalai, M. C. Eswariah, C. H. Chowdary, R. Kumar, M. Anusha, and K. Naresh, "Screening of thrombolytic activity of Bougainvillea glabra leaves extract by in-vitro," Asian Journal of Research in Pharmaceutical Science, vol. 2, pp. 134-136, 2012.

[54] S. K. Sherwani, M. M. Khan, A. Zubair, M. A. Shah, and S. Kazmi, "Evaluation of in vitro thrombolytic activity of Bougainvillea spectabilis leaf extract," International Journal of Pharmaceutical Sciences Review and Research, vol. 21, pp. 6-9, 2013.

[55] L. Arteaga Figueroa, L. Barbosa Navarro, M. Patiño Vera, and V. L. Petricevich, "Preliminary studies of the immunomodulator effect of the Bougainvillea x buttiana extract in a mouse model," Evidence-Based Complementary and Alternative Medicine, vol. 2015, Article ID 479412, 9 pages, 2015.

[56] E. V. Ikpeme, U. B. Ekaluo, O. U. Udensi, E. E. Ekerette, and M. Pius, "Phytochemistry and reproductive activities of male albino rats treated with crude leaf extract of great bougainvillea (Bougainvillea spectabilis)," Asian Journal of Scientific Research, vol. 8, no. 3, pp. 367-373, 2015.

[57] H. Saikia and A. Lama, "Effect of Bougainvillea spectabilis leaves on serum lipids in albino rats fed with high fat diet," International Journal of Pharmaceutical Sciences and Drug Research, vol. 3, pp. 141-145, 2011.

[58] B. Garg, N. M. Srivastava, and S. Srivastava, "Antihyperlipidemic effect of Bougainvillea glabra leaves in triton wr-1339 induced hyperlipidemic rats," Der Pharmacia Lettre, vol. 7, no. 7, pp. 187-190, 2015.

[59] G. Mandal, C. Chatterjee, and M. Chatterjee, "Evaluation of anti-inflammatory activity of methanolic extract of leaves of Bougainvillea spectabilis in experimental animal models," Pharmacognosy Research, vol. 7, no. 1, pp. 18-22, 2015.

[60] R. N. Okigbo, C. L. Anuagasi, J. E. Amadi, and U. J. Ukpabi, "Potential inhibitory effects of some African tuberous plant extracts on Escherichia coli, Staphylococcus aureus and Candida albicans," International Journal of Integrative Biology, vol. 6, pp. 91-98, 2009.

[61] P. Chauhan, S. Mahajan, A. Kulshrestha et al., "Bougainvillea spectabilis exhibits antihyperglycemic and antioxidant activities in experimental diabetes," Evidence-Based Complementary and Alternative Medicine, vol. 21, no. 3, pp. 177-185, 2015.

[62] J. Joshny, D. Ramya Devi, and B. N. Vedha Hari, "Phytochemical and in-vitro anthelmintic activity of hydro alcoholic extract of
Bougainvillea glabra," International Journal of Pharmacy and Pharmaceutical Sciences, vol. 4, no. 2, pp. 115-117, 2012.

[63] L. A. Figueroa, L. B. Navarro, M. P. Vera, and V. L. Petricevich, "Antioxidant activity, total phenolic and flavonoid contents, and cytotoxicity evaluation of Bougainvillea x buttiana," International Journal of Pharmacy and Pharmaceutical Sciences, vol. 6, no. 5, pp. 497-502, 2014.

[64] M. Medpilwar, D. Maru, M. Upadhyay, N. Lavania, M. Vernekar, and M. Harmalkar, "In-vitro antioxidant and anti-lipid peroxidation activity of ethanolic extracts of bougainvillea shubhra, bougainvillea peruviana and bougainvillea bhuttiana golden glow: A comparative study," Journal of Natural Remedies, vol. 15, no. 1, pp. 43-48, 2015.

[65] N. Sahu and J. Saxena, "Nitric oxide radical scavenging assay of Bougainivllea glabra, 'Choicy"' World Journal of Pharmacy and Pharmaceutical Sciences, vol. 3, pp. 397-401, 2014.

[66] K. V. Bhaskara Rao, H. Nidhi, D. Dipankar, D. Garima, G. Kumar, and L. Karthik, "Phytochemical profile, in vitro antioxidant property and HPTLC analysis of methanol extract of Bougainvillea glabra (Nyctaginaceae)," International Journal of Pharmaceutical Sciences Review and Research, vol. 31, no. 2, pp. 235-241, 2015.

[67] N. Naidu, R. Srilekhs, V. Pethuru, B. Anusha, E. Kejiya, and E. B. Lakshmamma, "Evolution of anti-microbial and anti-oxidant activity of alcoholic extract of Bougainvillea glabra flowers," Indo American Journal of Pharmaceutical Research, vol. 2, pp. 67-71, 2016.

[68] R. N. Venkatachalam, K. Singh, and T. Marar, "Bougainvillea spectabilis, a good source of antioxidant phytochemicals," Research Journal of Pharmaceutical, Biological and Chemical Sciences, vol. 3, pp. 605-613, 2012.

[69] S. Bhatia and M. L. Lodha, "RNase and DNase activities of antiviral proteins from leaves of Bougainvillea $x$ buttiana," Indian Journal of Biochemistry and Biophysics, vol. 42, no. 3, pp. 152-155, 2005.

[70] S. Narwal, A. Balasubrahmanyam, P. Sadhna, H. C. Kapoor, and M. L. Lodha, "A systemic resistance inducing antiviral protein with N-glycosidase activity from Bougainvillea $x$ buttiana leaves," Indian Journal of Experimental Biology (IJEB), vol. 39, no. 6, pp. 600-603, 2001.

[71] K. S. Rao, A. Nagaiah, G. D. Kumar, L. Saiprasanth, and R. D. Kumar, "Cardiotonic activity of aqueous flower extract of Bougainvillea glabra," International Journal of Research in Pharmacy and Chemistry, vol. 3, pp. 513-517, 2013.

[72] A. Lamien-Meda, C. Lamien, M. Compaoré et al., "Polyphenol content and antioxidant activity of fourteen wild edible fruits from Burkina Faso," Molecules, vol. 13, no. 3, pp. 581-594, 2008.

[73] Marvin, MarvinSketch 18.4, 2018, http://www.chemaxon.com.

[74] S. Jawla, Y. Kumar, and M. S. Y. Khan, "Hypoglycemic potential of Bougainvillea spectabilis root bark in normal and Alloxaninduced diabetic rats," Pharmacologyonline, vol. 3, pp. 73-87, 2011.

[75] B. N. Joshi, M. Bhat, S. K. Kothiwale, A. R. Tirmale, and S. Y. Bhargava, "Antidiabetic properties of Azardiracta indica and Bougainvillea spectabilis: In vivo studies in murine diabetes model," Evidence-Based Complementary and Alternative Medicine, vol. 2011, Article ID 561625, 9 pages, 2011.

[76] B. N. Joshi, M. Bhat, S. S. Zinjarde, S. Y. Bhargava, and A. R. Kumar, "Antidiabetic Indian plants: a good source of potent amylase inhibitors," Evidence-Based Complementary and Alternative Medicine, vol. 2011, Article ID 810207, 6 pages, 2011. 
[77] S. Jawla, Y. Kumar, and M. S. Y. Khan, "Hypoglycemic activity of Bougainvillea spectabilis stem bark in normal and alloxaninduced diabetic rats," Asian Pacific Journal of Tropical Biomedicine, vol. 2, no. 2, pp. S919-S923, 2012.

[78] U. M. Gómez Bache, L. Barbosa Navarro, and V. L. Petricevich, "Estudio preliminar del efecto hipoglucemico del extracto de Bougainivillea $x$ buttiana (variedad naranja) en modelo murino," Revista Mexicana de Ciencias Farmacéuticas, vol. 47, 2016.

[79] A. M. El-Mahmood and J. H. Doughari, "Bacteriological examination of some diluted disinfectants routinely used in the Specialist Hospital Yola, Nigeria," African Journal of Pharmacy and Pharmacology, vol. 3, no. 5, pp. 185-190, 2009.

[80] N. Mishra and V. L. Tandon, "Haematological effects of aqueous extract of ornamental plants in male swiss albino mice," Veterinary World, vol. 5, no. 1, pp. 19-23, 2012. 


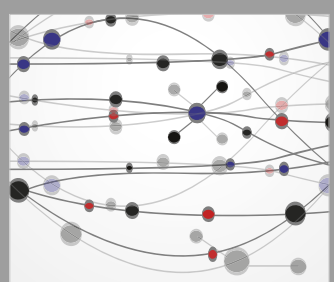

The Scientific World Journal
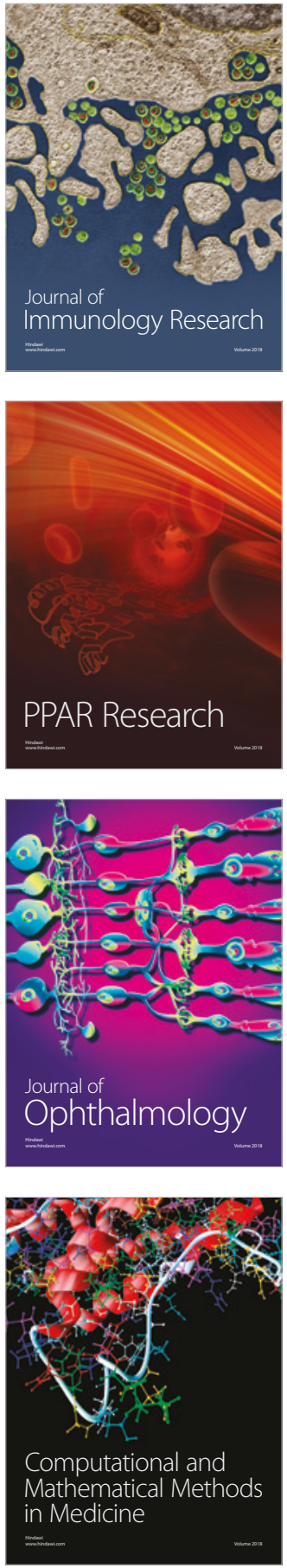

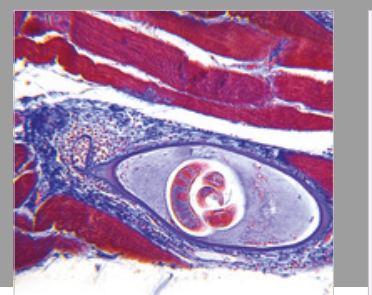

Gastroenterology Research and Practice

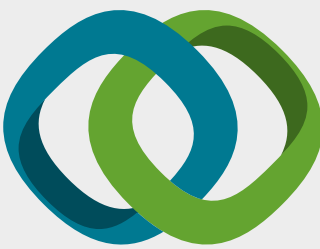

\section{Hindawi}

Submit your manuscripts at

www.hindawi.com
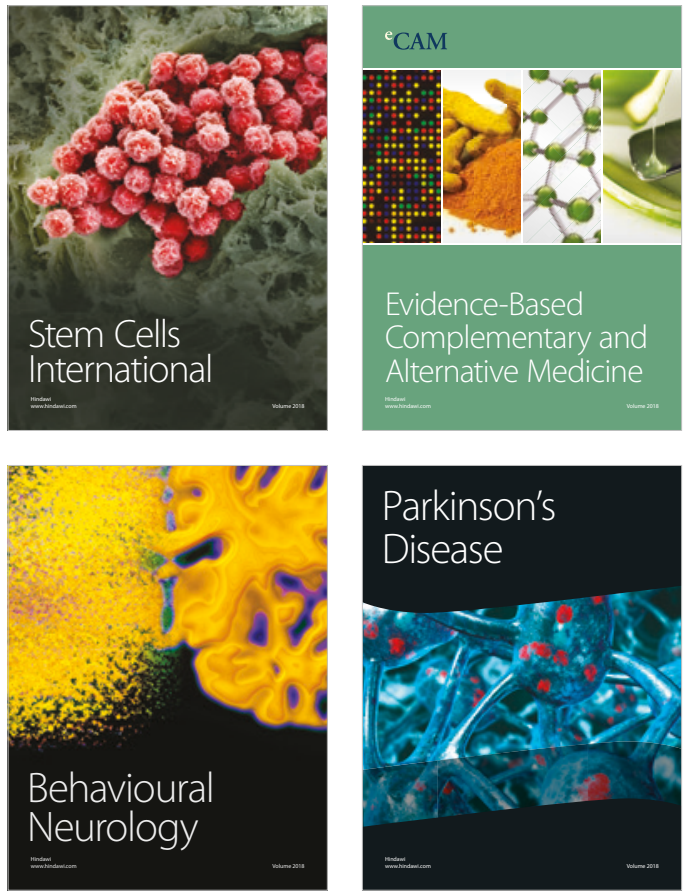

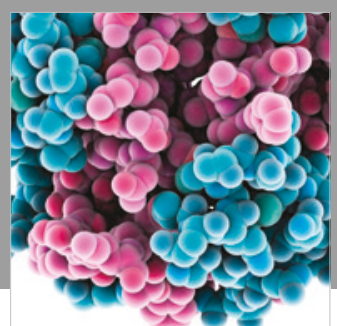

ournal of

Diabetes Research

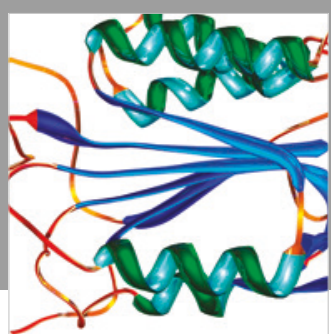

Disease Markers
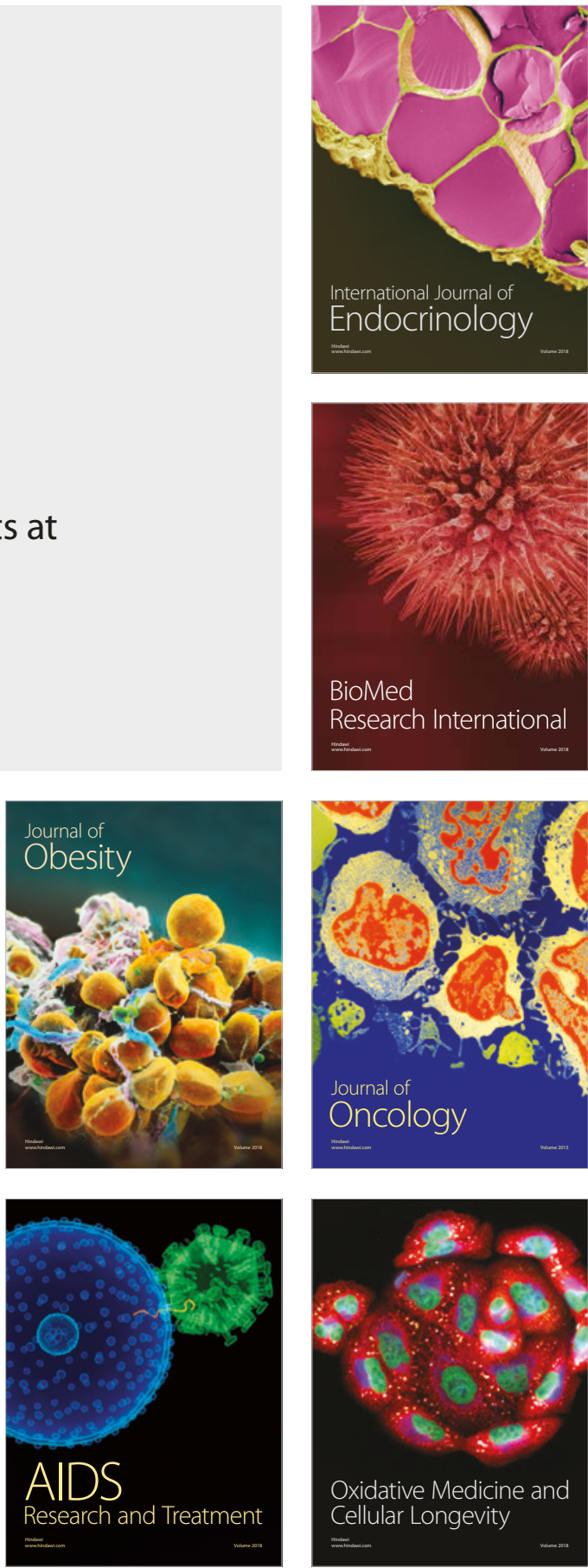